2018

\title{
Clustered Networks Protect Cooperation Against Catastrophic Collapse
}

Gwen Spencer

Smith College, gspencer@smith.edu

Follow this and additional works at: https://scholarworks.smith.edu/mth_facpubs

Part of the Mathematics Commons

\section{Recommended Citation}

Spencer, Gwen, "Clustered Networks Protect Cooperation Against Catastrophic Collapse" (2018). Mathematics and Statistics: Faculty Publications, Smith College, Northampton, MA.

https://scholarworks.smith.edu/mth_facpubs/38 


\title{
Clustered Networks Protect Cooperation Against Catastrophic Collapse
}

\author{
Gwen Spencer* \\ Smith College, Northampton, MA 01063, USA
}

\begin{abstract}
Assuming a society of conditional cooperators (or moody conditional cooperators), this computational study proposes a new perspective on the structural advantage of social network clustering. Previous work focused on how clustered structure might encourage initial outbreaks of cooperation or defend against invasion by a few defectors. Instead, we explore the ability of a societal structure to retain cooperative norms in the face of widespread disturbances. Such disturbances may abstractly describe hardships like famine and economic recession, or the random spatial placement of a substantial numbers of pure defectors (or round-1 defectors) among a spatially-structured population of players in a laboratory game, etc.

As links in tightly-clustered societies are reallocated to distant contacts, we observe that a society becomes increasingly susceptible to catastrophic cascades of defection: mutually-beneficial cooperative norms can be destroyed completely by modest shocks of defection. In contrast, networks with higher clustering coefficients can withstand larger shocks of defection before being forced to catastrophicallylow levels of cooperation. We observe a remarkably-linear protective effect of clustering coefficient that becomes active above a critical level of clustering. Notably, both the critical level and the slope of this dependence is higher for decision-rule parameterizations that correspond to higher costs of cooperation. Our modeling framework provides a simple way to reinterpret the counter-intuitive and widely-cited human experiments of Suri and Watts (2011) while also affirming the classical intuition that network clustering and higher levels of cooperation should be positively associated.
\end{abstract}

Keywords: cooperation, clustering, threshold models, social influence, contagion, repeated game-play.

\section{Introduction and Motivation}

The ubiquity of short average path lengths in social networks is often explained as reflecting the advantage of fast diffusion of information (starting from the foundational work of Granovetter (1973)). In graphs with low density, "weak ties" that reach to otherwise-distant members of the network are key to obtaining short average path lengths, giving rise to a "small world" property (Granovetter (1973); Watts \& Strogatz (1998)). A model in which all utility is gained through quick access to information would predict a strong preference for these longer ties. At the same time, observations of significant network clustering (which requires many short ties to neighbors of neighbors) are also ubiquitous in real data about social networks. In fact, cluster (or community) detection is a very active area of contemporary research, For example, see (Fortunato (2010)); (Leskovec et al. (2010)), and many others. What advantages might contribute to the popularity of short ties?

${ }^{*}$ Corresponding author: Gwen Spencer. Address: Burton Hall, Smith College. Northampton, MA 01063. Phone: 413-5853830. Fax: (413) 585-3786. gspencer@smith.edu. This research did not receive any specific grant from funding agencies in the public, commercial, or not-for-profit sectors. 
While some factors that encourage clustering are circumstantial (e.g. it is easier to meet friends of friends) there is also a recent interest in ways that social network clustering may itself be utility-providing. ${ }^{1}$ Moving away from well-mixed populations, extensive classical study in evolutionary biology and evolutionary game theory has posited that a stable (or semi-stable) interaction networks could promote cooperation among self-interested individuals. Nowak et al. (1994) conducted early computational work in lattices (see Nowak (2006) and Roca et al. (2009) for more comprehensive surveys). Sociologists have studied "outbreaks of cooperation" (Glance \& Huberman (1993)): could more-clustered network structure foster sudden transitions to widespread cooperation (Watts \& Strogatz (1998))? Since, "many of the benefits sought by living things are disproportionately available to cooperating groups" (Axelrod \& Hamilton (1981)) clustered social structure would then yield advantage, and might be interpretable as strategic.

We conduct a computational study to explore another source of utility that can stem from highly-clustered social networks. Suppose that a society starts from a state of widespread cooperation: how robust is this widely-cooperative equilibrium against a wide rash of defections? Consider acts of theft that are unlikely to be discovered. In times of plenty, the cooperative norm of not stealing in such cases may be comfortably maintained. In contrast, during times of pressing hardship (e.g., depression or famine), individuals may be increasingly likely to weigh the financial benefits of stealing above the (often non-monetary) benefits of adhering to a cooperative norm of not stealing. If thefts are no longer rare, and individuals are conditional cooperators or moody conditional cooperators, ${ }^{2}$ how will behavior evolve in the wider society?

This unobserved-theft motivation references a common game-theoretic interpretation of the decision to conditionally cooperate as the outcome of a cost-benefit calculation. In particular, each individual weighs a (usually fixed) cost of cooperation against a benefit of cooperation that is non-decreasing in the fraction of (nearby) cooperators. In economics, such a benefit function is said to exhibit a network effect. Our question about robustness of cooperative norms supposes that for various external reasons, the cost-benefit calculations of a sizable number of individuals is temporarily perturbed so that they choose to defect in circumstances where they might have typically cooperated. In addition to this abstract economic argument about how spontaneous temporary defections might emerge we mention several behavioral phenomena documented in repeated networked game play that could threaten long-term levels of cooperation in similar ways. First, in human experiments, the uncontrolled spatial placement of large percentages of round-1 defectors and even the pure defectors from Grujić et al. (2010) functions as a randomly-distributed shock to cooperation: can long-term cooperation thrive in the network despite these "bad actors" entering the game as defectors? Secondly, networked human experiments have documented exploration behavior (players test to gauge what rewards are available from temporarily switching to defection) (Traulsen et al. (2010)). Our question is whether such perturbations (or shocks) are less damaging to societies that are structured in special ways.

Can the structure of a society or organization protect against a complete collapse of cooperative norms? If so, what advantages might this afford? Societies that retain a stable cooperative core despite occasional modest cascades of defection may regrow widespread cooperative norms more easily and quickly, effectively making cooperative norms more resilient and leading to a higher stream of benefits over time. How does reallocation of local short links to long weak ties impact the ability of a social network to retain at least some cooperative foundation?

Relationship to Existing Literature. The "outbreaks of cooperation" or cooperation as a "social contagion" modeling framework draws on ideas from epidemiology, assuming that the society initially exists in a widely-uncooperative state before cooperative behavior arises (and begins to spread) (Fowler \& Christakis (2010); Centola \& Macy (2007)). This emphasis extends classical (pre-network) exploration of the initial viability of cooperative strategies (Axelrod \& Hamilton (1981)). In this framework, the question is whether cooperation (arising from a few acts of spontaneous altruism) can invade a society of defectors, and in

\footnotetext{
${ }^{1}$ Behaviors which are utility-providing may emerge as a result of conscious rational choice, or unconsciously as a result of some kind of evolutionary selection at the individual, family, or group level.

${ }^{2}$ They cooperate or contribute when others they observe also cooperate or contribute, as described in modeling work (Granovetter (1978); Glance \& Huberman (1993)), and empirical studies (Fowler \& Christakis (2010); Suri \& Watts (2011)). The moody variant also permits dependence on the player's own past actions (see, for example, Grujić et al. (2010), Gracia-Lázaro et al. (2012), Grujić et al. (2014)) and (Horita et al. (2017)).
} 
particular, how the social structure of the society might encourage or discourage such an invasion.

In contrast, behavioral studies seem to suggest that cooperation (arising locally) is a rather natural state for groups of humans. For example, empirical studies in behavioral economics show that humans learn to cooperate quite successfully when a game is played repeatedly in small groups (van Huyck et al. (1990); Knez \& Camerer (2000); Weber (2006)). In fact, even types of games that end divisively if played from a cold start can be played cooperatively by a group that has previous experience playing cooperatively together (Cason et al. (2012); Knez \& Camerer (2000)). Remarkably, cooperative play can even spill over into play with different groups of partners (Cason et al. (2012); Fowler \& Christakis (2010)). Experiments also indicate that, even for divisive weakest-link games (where payoffs are equal to the contribution of the stingiest contributor), large cooperative groups can be created by slowly adding new members to groups that have been observed successfully cooperating (Weber (2006)). Weber's experiments in (Weber (2006)) were motivated by the lack of prior lab-based evidence replicating the wide-scale cooperation plainly apparent in real-world observations: large groups like "firms and communities-where coordination plays a crucial role...have managed to coordinate successfully." Inverting the "outbreaks" modeling framework, consider that an extensive social network in a widely-cooperative state may emerge from long ties being formed between many small communities where cooperation arose (and stabilized) spontaneously, or by adding new members slowly to increase the size of successfully-cooperating groups. Once a large increasingly-connected cooperative society is formed, can this widespread cooperation be maintained?

Mirroring the classical study of how spontaneous acts of personal altruism might seed outbreaks of cooperation, both theoretical and empirical lines of reasoning highlight the plausibility of spatially-distributed acts of defection in the midst of a highly cooperative society. Above we've mentioned an abstract economic argument about how depression or famine might cause temporary shifts in the cost-benefit calculations of some members of a society (increasing the relative cost of cooperation, and thus introducing defections). We've also noted empirical observations of exploration strategies where a player occasionally chooses defection while surrounded by cooperating neighbors (an exploitation behavior observed in repeated Prisoner's Dilemma games), and the existence of substantial populations of players who enter controlled laboratory games by defecting in round 1 or even applying pure defection strategies for the duration of a repeated network game. Defections in the presence of widespread cooperation may even be unintentional. In the behavioral literature, researchers consider the situation of cooperative intentions being interrupted by inadvertent defections very plausible: extensive human experiments have explored how players respond when both a neighbor's intention (to cooperate) and a randomly-realized action are observed (Rand et al. (2015)). The finding is that subjects behave as if defections which arise without the malicious intent of the opposing player are less sanction-able. Given a broad range of mechanisms that might introduce many spatiallydistributed defections across a cooperative society, are some social structures better at avoiding catastrophic collapse of cooperation in the face of these large disturbances?

Our Contribution. Our computational study considers a fully-cooperative network of conditional cooperators that is hit by a randomly-spatially-distributed shock of defection. Under our basic Conditional Cooperation ( $C C$ ) Model individuals apply a widely-studied threshold decision rule to determine when to cooperate (as in (Granovetter (1978); Glance \& Huberman (1993); Watts \& Strogatz (1998); Watts (1999))). Simply, if an $h$-fraction of their neighbors cooperated in the previous time step, then the individual will cooperate in the next time step. If an individual is shocked with defection then they defect for a short period of time regardless of neighbor behavior. What is the long-term impact of such a defection shock on the society-wide level of cooperation? We focus on what size of defection shock is sufficient to cause catastrophic collapse of initially widespread norms of cooperation.

We repeat our analysis for a generalization of the basic CC model, the Heterogeneous Moody Conditional Cooperation (MCC) Model. This extended model was developed and refined across a growing body of compelling behavioral studies on repeated Prisoner's Dilemma (e.g. see Grujić et al. (2010), Gracia-Lázaro et al. (2012), Grujić et al. (2014)) and Public Goods Games (Horita et al. (2017)).

To avoid confusion, we comment explicitly on our purpose in the present study. Extensive behavioral studies have sought to clarify what decision rules humans use to play a variety of repeated network games. 
For example, Grujić et al. (2010) demonstrated that a heterogeneous MCC Model predicts human play of networked Prisoner's Dilemma much more faithfully than an "imitate-the-best" decision model. We take as given that different games (e.g. Prisoner's Dilemma vs. Public Goods Games) and different specifics of game presentation and reward structure can impact the form of player decision rules, and the parameter values of observed decision rules. Further, an extremely interesting body of work has begun to expose how reinforcement learning may be the driving mechanism behind the phenomena of Moody Conditional Cooperation decision rules (e.g. Horita et al. (2017)). In contrast, our interest in the present study is to understand what implications the widely-studied basic CC and heterogeneous $M C C$ decision rules have for possible advantages available to specially-structured societies. Our experimental framework could certainly be applied to other decision rules of interest in the future (for example, the stochastic strategy updating suggested by the human studies of Traulsen et al. (2010)).

In Sections 3 and 5, using the random-rewiring procedure of Watts \& Strogatz (1998), we study Conditional Cooperation (CC) and Moody Conditional Cooperation (MCC) across a "continuous" series of networks. Community structure is gradually eroded as links are reallocated from dense local communities to information-seeking long ties. We comment on key observations that appear remarkably consistent across several synthetic networks and a sizable real-network example.

- A Protective Effect of Clustering. Highly-clustered networks can provide protection of cooperative norms from catastrophic collapse caused by a randomly-distributed shock of defections in the network. Nevertheless, some defection shocks are large enough to cause catastrophic collapse across topologies: pushed into catastrophic collapse by severe defection shocks, the behavior of topologies will be very difficult to distinguish.

As links are reallocated from local communities, we observe a surprisingly smooth decreases in the size of shock the network can endure before catastrophic collapse of cooperation. A society with an increasing prevalence of long ties appears to foster large-scale cascades of defection, even under milder initial defection shocks.

- The Protective Effect Only Becomes Active Above a "Critical Level of Clustering." For Conditional Cooperation, the clustering coefficient at which a protective effect of clustering becomes active consistently appears to increase with threshold $h$. When players apply high thresholds for cooperation, the protective effect of clustering only becomes active at quite high levels of clustering. Experiments for Moody Conditional Cooperation exhibit a similar "critical level" that increases as a heterogeneous population of players is increasingly composed of Stingy players.

When many players behave as if the cost of cooperation is high, a protective effect of clustering is predicted only at higher levels of clustering.

- Under Very "Low Cost of Cooperation" No Protective Effect of Clustering is Predicted. For Conditional Cooperation, when players apply low thresholds $h$, and for Heterogeneous Moody Conditional Cooperation, when many players behave very generously (cooperating despite high rates of neighbor defections), we observe no protective effect of clustering.

That is, the parameter values of local decision rules applied by players (in response to game presentation and reward structure) determine whether variation in topology has a predicted impact on long-term rates of cooperation in the network.

Our study as community structure is "smoothly eroded" via random rewiring has some conceptual similarities to how Garcia \& Vega-Redondo (2015) model the evolution of altruism as a function of levels of local cohesion of a population that is uniformly distributed on a one-dimensional space. Roughly speaking, local cohesion describes the fraction of within-group connections. Our second finding (that a critical level of clustering must be reached to activate a protective effect of clustering) may be interpretable as a message about regime change in the local-cohesion parameter space: only above a certain level of local cohesion is a protective effect of clustering predicted. Like their study, our results highlight that the initial conditions that fall in 
the "basin of attraction" of catastrophic collapse of cooperation depends critically on the cost of cooperation. ${ }^{3}$

A Novel View of Several Human Experiments. Our model can be used to reinterpret an empirical study that is often cited to argue against the importance of clustering towards higher levels of cooperative behavior. Suri \& Watts (2011) cite theoretical predictions of a cooperation-promoting effect of clustering as the motivation for their human experiments. The human experiments of Suri and Watts (Suri \& Watts (2011)) failed to find significant variation in cooperation levels when a repeated public-goods games was played across five network topologies of widely-varying clustering coefficient. Such an effect had been predicted on a qualitative basis in classical papers, so this experimental evidence appeared quite counter-intuitive when published.

In Section 4, we interpret the Suri and Watts experimental results in our modeling framework: because of the large fraction of human defectors in the first round of their laboratory game whose spatial placement in the network was not controlled, our model predicts that the experimenters should observe little-to-no significant variation among the varying network topologies studied. We argue that the major observations from the Suri and Watts experiments are not incompatible with either conditional cooperation or an important association between high clustering and cooperation: their observations are anticipated (across the studied topologies) by our simple threshold-for-cooperation model with threshold of $4 / 5$, and initial defection shock of $45 \%$. The proportion of initial defectors for the Suri and Watts public-goods game (modeled now as a randomlyspatially-distributed defection shock) appears to have simply been too large to be overcome by variation in social-network structure.

That is, perhaps the experimental design of Suri and Watts simply tested the "wrong" part of the parameter space where no impact of topology was predicted. In a systematic study on ring-topologies of varying degree, Rand et al. (2014) make a similar point: for some relationships of costs of cooperation and node degree, no effect of network variation is predicted, and an experiment with such cost/reward parameters should be doomed to fail. Under other costs of cooperation, however, an impact of topology is predicted, and conducting a human study with such parameters, Rand et al. (2014) actually do empirically detect an impact of topology.

Some prominent human studies focused on Moody Conditional Cooperation (MCC) are also cited to argue that network topology doesn't impact rates of cooperation. However, in addition to documenting high percentages of first round defectors (as we highlight for Suri \& Watts (2011)), many of these studies focus on a small number of networks where each has low clustering (well below 0.5). A comparative survey of human MCC studies (Grujić et al. (2014)) acknowledges that clustering may play a role, but points out that this network feature has not been systematically explored.

For example, Grujić et al. (2010) considered a degree-8 lattice (each ego node is surrounded by a "Moore Neighborhood") with clustering coefficient of only 0.43 (and nothing resembling dense community structure). In fact, Gracia-Lázaro et al. (2012) announced emphatically that network topology is irrelevant for cooperation after testing only two different networks, where both networks had low clustering coefficient. Due in part to the complex logistics and cost of conducting large human experiments, Gracia-Lázaro et al. (2012) test only two networks: the degree-4 lattice with periodic boundary conditions (clustering coefficient of 0 ), and a single alternate network with scale-free degree distribution. Based on the degree distribution and scale-free network visualization provided in Gracia-Lázaro et al. (2012) ${ }^{4}$ we estimate that the clustering coefficient of their scale-free network is at most 0.4. Gracia-Lázaro et al. (2012) observe cooperation collapse in both the degree-4 lattice and their particular scale-free network, and boldly title their work, "Heterogeneous networks do not promote cooperation when humans play a Prisoner's Dilemma."

Our computational exploration of Hetrogeneous Moody Conditional Cooperation in Section 5 provides several insights about why the cooperation collapse Gracia-Lázaro et al. (2012) observe for both their lowclustering networks may be a completely predictable outcome in the portion of the parameter space tested by

\footnotetext{
${ }^{3}$ In some sense, this cost can be qualitatively reverse-engineered from the decision rules applied by players.

${ }^{4}$ Precise construction information was not included either in the body or SI section of Gracia-Lázaro et al. (2012). The long paths of degree 2 in their scale-free network visualization (their Figure 1) don't appear consistent with the common preferential attachment method. We note that, given a fixed degree distribution, many different networks with widely-varying hierarchical and community structure can be constructed.
} 
their experimental design. In particular, they document a sizable 35-40\% shock of round-1 defections (their Figure 2), and large populations of very-stingy players (roughly $40 \%$ based on the right-hand intercepts of panels A and B of their Figure 3). Our framework suggests that the experimental findings of Gracia-Lázaro et al. (2012) (namely, cooperation collapse for both networks they study) are not incompatible with the existence of a strong protective effect of clustering in other portions of the parameter space for networked Heterogeneous $M C C$.

\section{Methods}

First we give precise statements of the conditional-cooperation spread models we that we will study.

\subsection{Basic Conditional-Cooperation Model}

Input: $G=(V, E)$ is an undirected graph. Each node $v \in V$ has a given fractional threshold for cooperation, $h_{v}$. At each timestep $t \in\{0,1,2, \ldots\}$, the function $c_{t}(v)$ describes whether node $v$ is cooperating or defecting:

$$
c_{t}(v)= \begin{cases}1 & \text { if } v \text { cooperates at time } t \\ 0 & \text { otherwise }\end{cases}
$$

Update Dynamics: At each timestep $t$, each node $v \in V$ updates its behavior depending on the behavior of $v$ 's neighbor set, $\delta(v)$, in the previous time step:

$$
c_{t+1}(v)= \begin{cases}1 & \text { if } \sum_{u \in \delta(v)} c_{t}(u) \geq h_{v} *|\delta(v)| \\ 0 & \text { otherwise. }\end{cases}
$$

Shock Treatment: Let $d$ denote the duration of the shock, and let $s$ denote the fraction of nodes to be shocked. A subset of nodes $D$, with $|D|=\lceil s *|V|\rceil$, is selected uniformly at random from $V$. For $u \in D$, and $i \in\{0,1, \ldots, d\}$, the value of $c_{i}(u)$ is forced to $0 .^{5}$ All non-shocked nodes, $v \in V \backslash D$, have $c_{0}(v)=1$.

Measurement of Treatment Effect: We simulate update dynamics until the convergence ${ }^{6}$ of $\sum_{v \in V} c_{t}(v)$. Letting $C$ denote the "Catastrophic-Collapse Threshold" we say cooperation has catastrophically collapsed if

$$
\sum_{v \in V} c_{t}(v)<C *|V|
$$

\subsection{Extended Model: Heterogeneous Moody Conditional Cooperation}

While the basic conditional cooperation model described above has been studied for many years, a compelling recent series of behavioral experiments on repeated Prisoner's Dilemma (e.g. see Grujić et al. (2010), GraciaLázaro et al. (2012), Grujić et al. (2014)) and additionally Public Goods Games (Horita et al. (2017)) on networks has exposed that human behavior can be more complex. In particular, choices to cooperate or defect (or to contribute generously vs. stingily to a public good) may depend both on the context faced by a player (how many of his neighbors cooperated at time $t$ ) and on the player's own actions at $t$. This phenomena was termed Moody Conditional Cooperation in Grujić et al. (2010). In particular, given fixed behavior of his neighbors, a player may be more inclined to cooperate at $t+1$ if he cooperated at $t$ (his past action putting him in a cooperative mood). Conversely, if the player defected at $t$ (putting him in a defective mood) then he may respond to the same fixed behavior of his neighbors at $t$ with defection at $t+1$.

Furthermore, many such experiments document that populations of players can appear quite heterogeneous in their decision rules. For example, Grujić et al. (2010) identifies 5 different player types. At the

\footnotetext{
${ }^{5}$ After $d$, nodes $u \in D$ resume normal updating.

${ }^{6}$ Under this model, there theoretically exist instances where convergence is to an oscillation between two values, but this is highly rare in practice.
} 
extremes are sizable populations of players who are either pure defectors (they always defect regardless of neighbor behavior) or pure cooperators (they always cooperate regardless of neighbor behavior). Remarkably, in a second experiment (after players have already played one full set of rounds), Grujić et al. (2010) find that $19.5 \%$ of players act as pure defectors. We note that the spatial placement of these pure defectors is not controlled and is highly similar to our shock treatment model feature where the shock has indefinite duration.

We apply a shock treatment and measurement of treatment effect in the same way as described for the basic conditional-cooperation model above. Using the notation introduced above, the input and update dynamics are generalized to the heterogeneous moody conditional-cooperation setting as follows.

Moody Input: $G=(V, E)$ is an undirected graph. Each node $v \in V$ has two given fractional thresholds for cooperation: $h_{v}^{c}$ (a cooperative-mood threshold for cooperation) and $h_{v}^{d}$ (a defective-mood threshold for cooperation).

Moody Update Dynamics: Each node $v$ updates its behavior depending both on the behavior of $v$ 's neighbors in the previous time step, and depending on $v$ 's own behavior at the previous time step:

$$
\begin{aligned}
& \text { If } c_{t}(v)=1 \text {, then } c_{t+1}(v)= \begin{cases}1 & \text { if } \sum_{u \in \delta(v)} c_{t}(u) \geq h_{v}^{c} *|\delta(v)|, \\
0 & \text { otherwise. }\end{cases} \\
& \text { If } c_{t}(v)=0 \text {, then } c_{t+1}(v)= \begin{cases}1 & \text { if } \sum_{u \in \delta(v)} c_{t}(u) \geq h_{v}^{d} *|\delta(v)|, \\
0 & \text { otherwise. }\end{cases}
\end{aligned}
$$

We note that, based on empirical evidence, we might generally assume that $h_{v}^{c} \leq h_{v}^{d}$. That is, when $v$ has cooperated at $t$ (and is in a cooperative mood), $v$ may be satisfied by observing $k$ neighbors cooperating at $t$ and then choose to cooperate at $t+1$, so that $c_{t+1}(v)=1$, while for the same player $v$ in a defective mood (having defected at $t$ ), observing $k$ neighbors cooperating at $t$ may fail to persuade $v$ to cooperate at $t+1$, so that $c_{t+1}(v)=0$.

In our formalism, it is easy to capture the pure cooperators and pure defectors from Grujić et al. (2010): pure cooperators simply have $\left(h_{v}^{c}, h_{v}^{d}\right)=(0,0)$ while pure defectors may have, for example $\left(h_{v}^{c}, h_{v}^{d}\right)=(1.1,1.1)$.
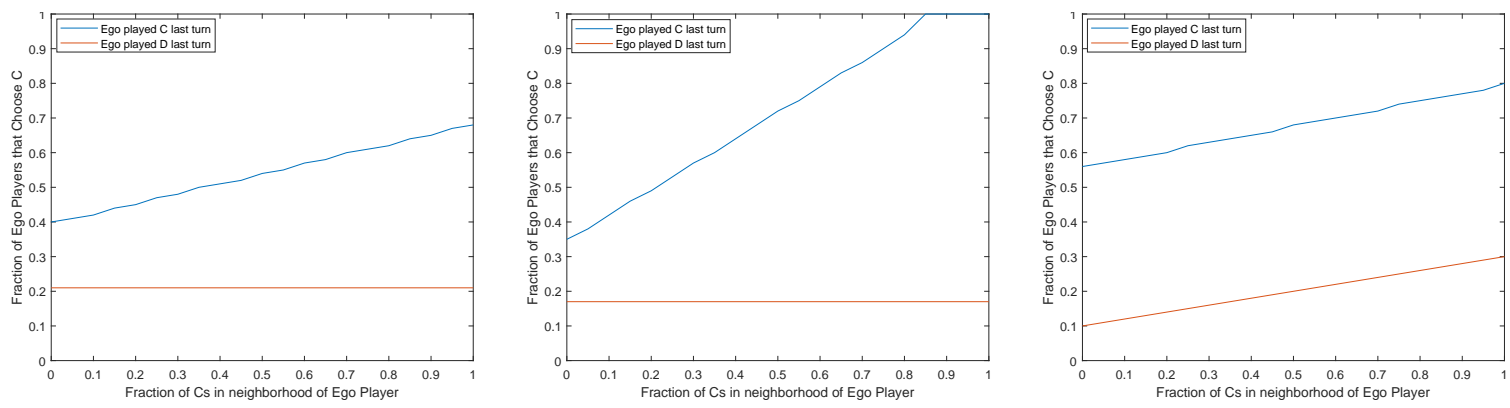

Figure 1: Heterogeneous Distributions Over $\left(h_{v}^{c}, h_{v}^{d}\right)$ Players Can Simulate a Range of Empirically-observed Moody Conditional Cooperation Responses. Panels are each generated by a different distribution over players, and quite-closely simulate the decision rules observed empirically in Figure 2 of Grujić et al. (2010) (two leftmost panels above) and Figure 3 from Horita et al. (2017) (rightmost panel above). Arbitrary combinations of intercepts and (non-negative) slopes for the cooperative mood response and the defective mood response can be easily captured by our modeling framework.

To make visually clear the connection between our formalism and observations from empirical behavioral 
studies, Figure 1 demonstrates how varying distributions over vectors of type

$$
\left[\begin{array}{l}
h_{v}^{c} \\
h_{v}^{d}
\end{array}\right]
$$

for the nodes of $V$ gives rise to a range of decision rules highly similar to those observed in the human experiments from Grujić et al. (2010), Gracia-Lázaro et al. (2012), Grujić et al. (2014)) and (Horita et al. (2017)), etc. As noted in the insightful analysis of Grujić et al. (2014) that compares several studies that document moody conditional cooperation, the variety of observed slopes and intercepts of similar plots from other human experiments are likely a response to the particular presentation and reward structure of the game, and even may depend on the players' level of prior experience with the game (a compelling finding from Grujić et al. (2010)). We do note that the slightly-negative slopes sometimes observed for players in defective moods (indicating exploitative behavior) are not captured by our formalism.

To motivate our experimental design, described explicitly in Section 5, we highlight that some features of the panels from Figure 1 are attributable to moodiness while others are driven by heterogeneity of the population of players. The vertical separation between cooperative mood response (shown in blue) and defective mood response (shown in red) is due to moodiness.

Next, consider the intercepts in Figure 1. In the leftmost panel, an intercept of roughly 0.2 for the defective mood response indicates that, for $20 \%$ of the players, even if they defected at $t$ and every one of their neighbors defected at $t$, they will still choose to cooperate at $t+1$. An intercept of 0.4 for the cooperative mood response indicates that, for $40 \%$ of the players, if they cooperated at $t$, even though all their neighbors defected at $t$, they will choose to continue cooperating at $t+1$. That is, a large percentage of players observed by Grujić et al. (2010) are remarkably generous in their choice to cooperate at $t+1$, behaving as if they view the cost of cooperation to be very low.

On the other hand, for the same leftmost panel of Figure 1, the value of the the cooperative mood response when all of a player's neighbors cooperate at $t$ is roughly 0.7 . That is, fully $30 \%$ of players, despite being in a cooperative mood, and observing every one of their neighbors cooperating at $t$, will change to a defection strategy at $t+1$. A large percentage of players observed by Grujić et al. (2010) are remarkably stingy, behaving as if they view the cost of cooperation to be very high.

Within our exploration of heterogeneous moody conditional-cooperation, we hope to avoid presenting an overwhelming number of population distributions. The 11 distributions we test are described explicitly at the beginning of Section 5, and are designed to vary gradually. Our objective in this design is that departures in catastrophic collapse behavior should be somewhat interpret-able. We hope that the tests we present will motivate further theoretical and computational examination of the implications of this more behaviorally-realistic space of decision rules.

\subsection{Reallocating local links to long ties}

Our first set of experiments considers a class of synthetic networks obtained by randomly-rewiring dense local communities (small complete graphs). As in (Watts \& Strogatz (1998)), we start from an initial network, and "rewire" each edge of the network with probability $p$. In particular, for each edge $e \in E$, with probability $p$ we rewire it as follows. First, randomly choose one of $e$ 's endpoints to be retained, then choose an alternative second endpoint from $V$ uniformly at random. Larger values for rewiring probability lead to decreased clustering coefficient of the network. ${ }^{7}$ By increasing $p$ gradually, we erode the community structure of $G$ in a somewhat "smooth" manner.

Our synthetic examples initialize this rewiring procedure with a set of small complete graphs. ${ }^{8}$ We will report results both for cases where the small communities have uniform sizes, and where these small community sizes are chosen from a normal distribution (included in the Appendix). We find consistent results

\footnotetext{
${ }^{7}$ We compute the clustering coefficient in the standard way. For each node $v$ in $G=(V, E)$, compute the ratio of the number of edges between neighbors of $v$ and the number of edges a complete graph on the neighbors of $v$ would contain. Average this ratio over all nodes in $G$ to obtain the clustering coefficient for the whole graph $G$.

${ }^{8}$ This roughly replicates a sequence of stochastic block models in which average degree is maintained.
} 

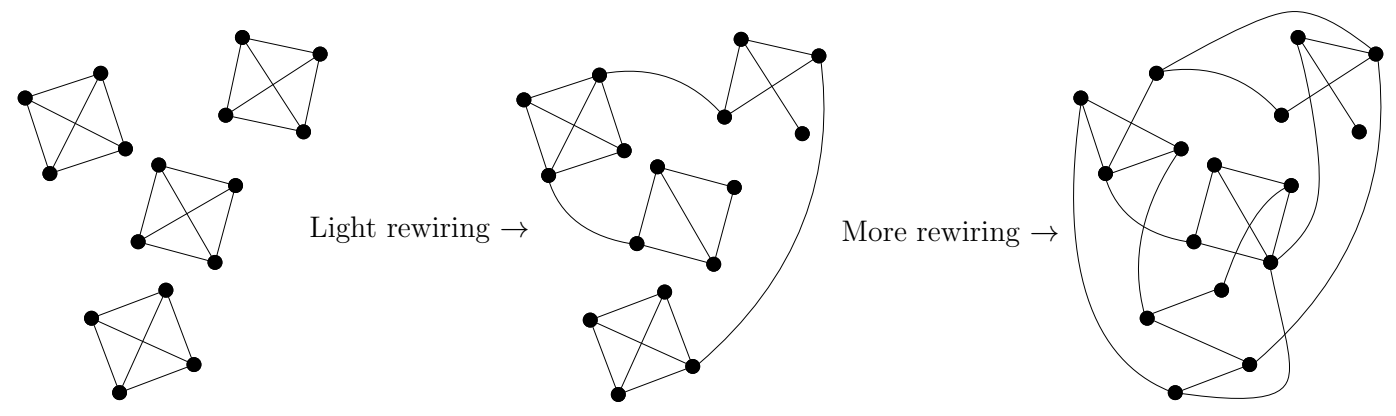

Figure 2: Schematic of Random Rewiring of Dense Local Communities (Complete Graphs). Clustering coefficient decreases gradually as the probability of rewiring, $p$, increases. This schematic depicts rewiring of four initial communities of size 4. Clustering coefficients left to right are: 1, 0.7, and 0.31. An additional impact of rewiring is visible here: $G$ 's degree distribution changes, in this case becoming non-uniform.

for both cases. For a real network data set on Co-boardmembership in Norway involving 1,421 individuals, we perform a similar gradual rewiring procedure, and also observe strikingly-similar behavior.

For each network, at each level of random rewiring, we consider the long-term cooperation at a range of increasing random-shock sizes: we focus on the size of random shock sufficient to push the network into catastrophic collapse of cooperation. Throughout our experiments, we regard cooperation to be in a state of catastrophic collapse if (after the period of shock ends) cooperation converges to less than $15 \%{ }^{9}$ Notice that the sufficient shock size to pass this boundary is a random variable that depends both on the realization of the rewired network, and on the placement of the random shock in the network.

All simulations were implemented in MATLAB running on a standard desktop computer.

\section{Results: Basic Conditional Cooperation}

Throughout this section, nodes apply a uniform threshold for cooperation, $h$. We examine 5 possible thresholds for cooperation: $h$ is in $\{0.5,0.6,0.7,0.8,0.9\}$. In this section we consistently consider a shock of duration $d=6$.

After this six-step shock duration, for networks of 50 nodes we almost always observed convergence within 5 additional time steps (at all thresholds tested). For networks of 200 nodes we noted somewhat rare instances that required up to 10 additional time steps (the vast majority still converged within 5 time steps post-shock). For our real-world network (1,421 nodes) convergence was almost always reached within 10 additional time steps (very rare instances required 20 time steps post-shock), and convergence appeared to occur more rapidly when thresholds were higher. Convergence times appeared similar for our Moody Conditional Cooperation tests in Section 5.

\subsection{Rewiring Dense Communities of Uniform Size}

Our first experiment considers an initial society composed of 5 complete graphs of 10 nodes each.

Figure 3 depicts how the size of a defection shock required to cause catastrophic collapse of cooperation depends on the probability of rewiring $p$. The five lines plotted in Figure 3 correspond to 5 possible thresholds. Confidence intervals depicted show two sample standard deviations on each side of the mean. All confidence intervals in the paper are computed based on sample size of 100.

As local links are rewired with higher probability, the size of shock required to push cooperative behavior to catastrophic levels ( $15 \%$ or less) decreases in a surprisingly smooth linear manner. The society becomes progressively less able to retain cooperation in the face of randomly-distributed shocks of defection. This

\footnotetext{
${ }^{9}$ The choice of $15 \%$ is somewhat arbitrary: in the appendix we include versions of many figures for an alternative definition of catastrophic collapse of $30 \%$. The trends are very similar.
} 


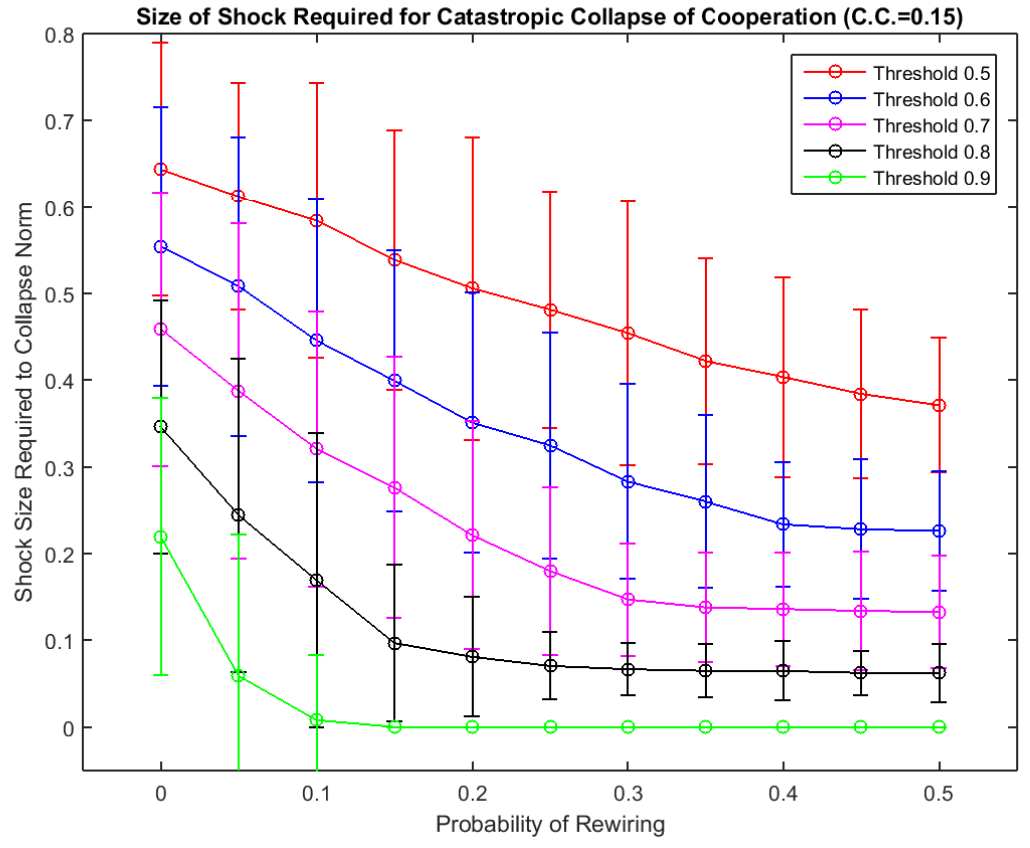

Figure 3: Rewiring Reduces Ability to Withstand Defection Shocks. Five initial communities of ten individuals each.

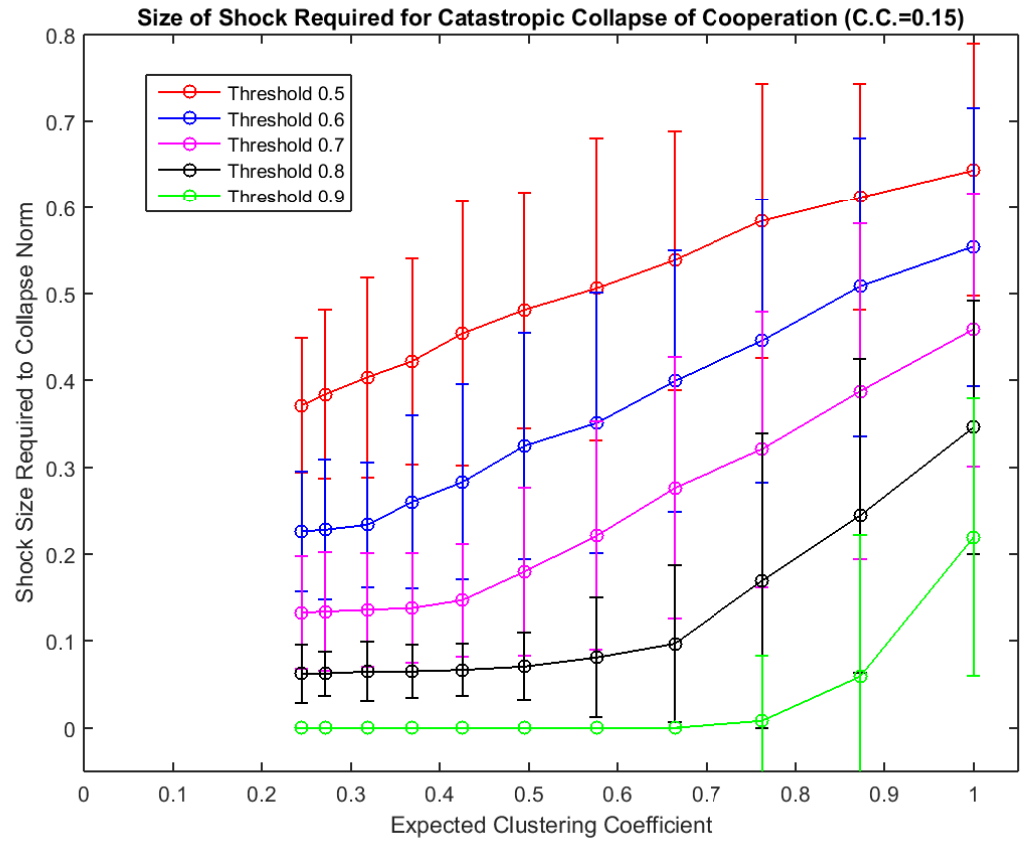

Figure 4: High Clustering Increases Ability to Withstand Defection Shocks. Five initial communities of ten individuals each. 

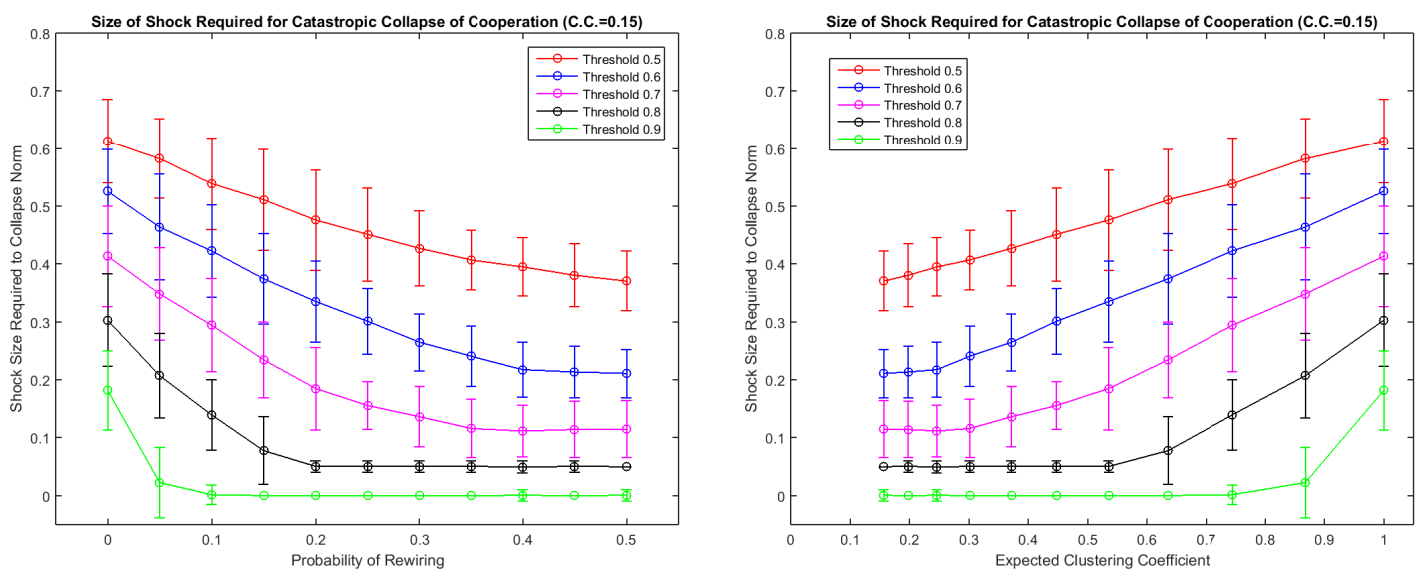

Figure 5: Ability to Withstand Defection Shocks vs. Rewiring (left panel) and Clustering Coefficient (right panel). Twenty initial communities of ten individuals each (total society of 200 nodes).

appears to hold for each uniform threshold in $\{0.5,0.6,0.7,0.8,0.9\}$. As rewiring increases, particularly at the higher thresholds ( 0.8 and 0.9$)$, we observe that even modest levels of rewiring have caused such a steep decrease in ability to withstand shocks that a kind of "bottoming out" is observed: there is a rewiring probability above which the defection shock size required to cause catastrophic collapse stabilizes.

As rewiring probability increases, the clustering coefficient decreases. In Figure 4 we depict the same data from Figure 3 as a function of the expected clustering coefficient for the rewired graph.

Consider Figure 4. After an initial period where the shock required to cause catastrophic collapse is stable, we observe a remarkably-linear-looking increasing protective effect as clustering coefficient increases. Notably, the clustering coefficient at which this protective effect becomes active increases with the threshold for cooperation applied by nodes. For example, when nodes apply threshold for cooperation 0.6 a linear protective effect appears to become active as clustering passes 0.35 , whereas for threshold 0.8 this protective effect isn't active until clustering passes roughly 0.75. Interpreting this trend: when nodes apply high thresholds-for-cooperation to the behavior of their neighbors, our model predicts that networks with a wide range of clustering coefficients will be forced into catastrophic collapse by a modest shock of defection. In such cases, the long-term behavior of such networks will be difficult to distinguish. This point will be central in considering the human experiments from (Suri \& Watts (2011)).

Running the same experiment in a larger initial society of 20 communities of 10 individuals each, we obtain Figure 5. Qualitatively the behavior appears very similar to Figures 3 and 4 even though the relative size of the small initial communities and the total society is very different $(1 / 20$ th vs. $1 / 5$ th). Further, we observe very similar behavior under alternate choices for the shock duration (rather than the $d=6$ we use as a standard in this section and Section 5). See the Appendix for these supplemental figures.

Our results in Figures 3, 4, and 5 are remarkably consistent. To verify that this consistency is not an artifact of our choice that initial communities have uniform size of 10 individuals, we conducted the same analysis when the initial community sizes were realized randomly from a normal distribution. We tested several parameter combinations (described in Table 1 below). Our results were very similar. Figures from these supplemental tests are included in the Appendix. 


\begin{tabular}{c|c|c} 
Total society & Mean Community Size & St. Dev. of Community Size \\
\hline 50 & 10 & 5 \\
200 & 10 & 5 \\
200 & 20 & 5
\end{tabular}

Table 1: Non-uniform Initial Community Sizes Produce Highly Similar Findings. See Appendix for corresponding figures.

\subsection{Rewired Norwegian Co-boardmembership Network}

Our results for synthetic networks suggest that in networks with high clustering (but low overall density), reallocating local links to long random ties results in a decrease in the network's ability to withstand large random shocks of defection. Will such a trend occur in data sets describing real patterns of human interaction?

To examine the trend across the range of clustering coefficients, we test in a real network data set with very high clustering. We consider a network created in (Seierstad \& Opsahl (2011)) based on boards of public limited companies in Norway: each individual is represented by a node, and two nodes are connected if the corresponding individuals serve on a common board. As a result of this construction method, the network is composed of a number of small complete subgraphs (one corresponding to each board) that may overlap in multiple members. As a result, the initial form is somewhat similar to the synthetic examples we constructed, with a significant departure.

Figure 6 depicts the degree distribution for the Norwegian Co-boardmembership network. In contrast with our synthetic examples that have binomial-like degree distribution (due to their construction), the Norwegian Co-boardmembership degree distribution appears power-law-like (or scale-free-like). This scalefree degree distribution shape is considered to be typical of many real social network data sets.

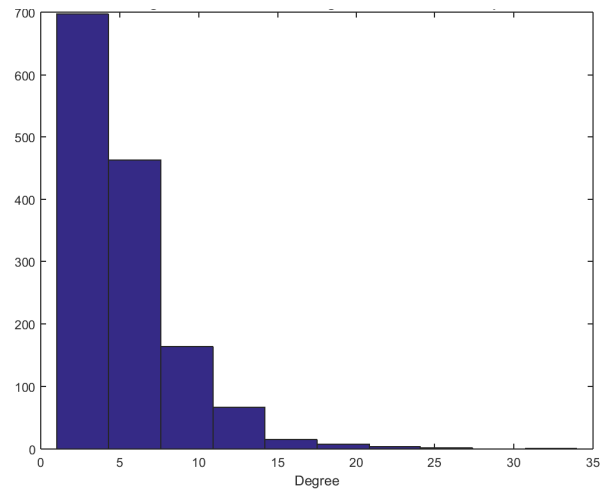

Figure 6: Degree Distribution for Norwegian Co-boardmembership Network (1,421 nodes).

Figure 7 shows our experimental results for the Norwegian Co-boardmembership network. As in the synthetic networks generated earlier, we observe a linear-looking protective effect of clustering that becomes active at (and above) a critical value of the clustering coefficient. This critical value appears to increase as a function of the threshold for cooperation applied by each node. Findings are milder but qualitatively similar for a definition of catastrophic collapse of 30\%-cooperation-or-less (see the Appendix for this alternate version of Figure 7).

\subsection{Basic Conditional Cooperation When Thresholds are Very Low}

Figures 3 - 7 focus on shock sizes that cause catastrophic collapse for thresholds $h$ from $\{0.5,0.6,0.7,0.8,0.9\}$. In the interval of clustering coefficients where a protective effect of clustering is observed, it appears that the 

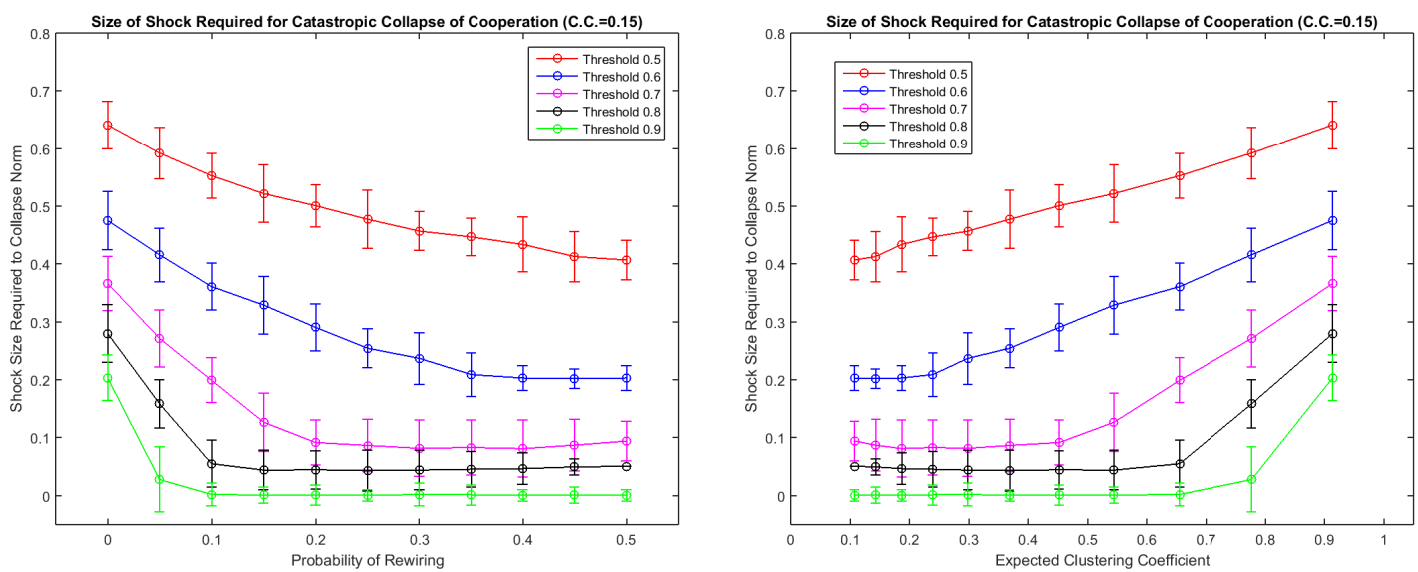

Figure 7: Ability to Withstand Defection Shocks vs. Rewiring (left) and Clustering Coefficient (right). Norwegian Co-board-membership Network (1,421 nodes).

slope of this effect is higher when $h$ is higher. For example, consider the right-hand panel of Figure 5. When $h=0.8$ a protective effect of clustering is observed above a critical level of 0.65 , in the range $[0.65,1]$. The slope of this protective effect is quite steep when compared to the slope of the protective effect of clustering observed for threshold $h=0.5$ over the longer range $[0.15,1]$.

Suppose that the uniform threshold is decreased from 0.5: Figure 8 shows that the protective effect of clustering will continue to become more shallow as $h$ decreases, vanishing completely for the lowest thresholds.
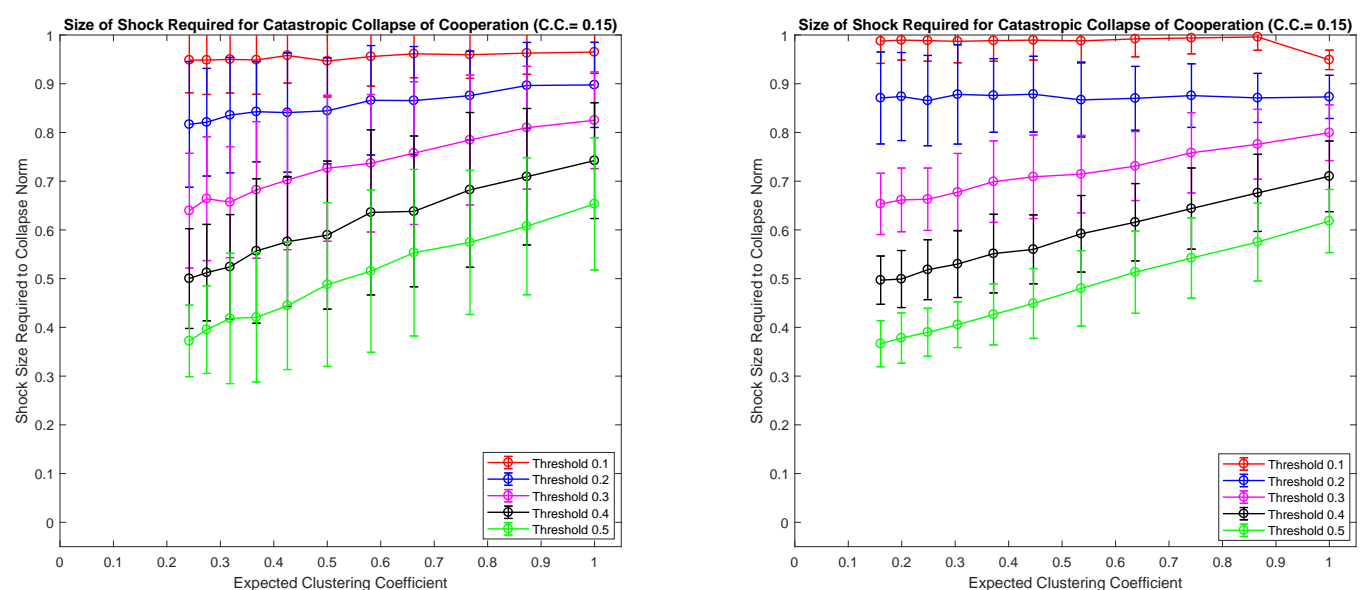

Figure 8: At the Very-Lowest Thresholds, No Protective Effect of Clustering is Observed. Left Panel: Five initial communities of ten individuals each. Right panel: Twenty initial communities of ten individuals each.

Qualitatively, when players respond as if the cost of cooperation is very low (that is, even a small number of cooperating neighbors are sufficient to persuade the focal individual to cooperate), cooperation is so resilient that clustered network structure can't yield improvements over more randomly-structured networks. We draw special attention to this behavior under "very generous" conditional cooperators: our analysis of the heterogeneous Moody Conditional Cooperation Model in Section 5 will also show that diluting a more demanding population with generous players can suppress the impact of network topology. 


\section{Interpreting the Suri and Watts experiments in terms of defec- tion shock and likelihood of catastrophic collapse}

In 2011, Suri and Watts published a highly-cited empirical study that appeared to discredit the classicallypredicted advantage of network clustering to aid cooperation. We demonstrate how our simple model can be parametrized to anticipate the catastrophic collapse they observe across topologies. We argue that the observations in (Suri \& Watts (2011)) can be understood as specific to a portion of the parameter space, and not as a general evidence against the role of clustered topology in encouraging cooperation.

Background on (Suri \& Watts (2011)). To explore the traditional reasoning that wide-scale human cooperation is reinforced when humans interact with more stable groups of contacts, Suri and Watts conducted a series of web-based human experiments.

Twenty-four players were arranged as nodes in different network topologies and player payoffs for each round of a public-goods game depended on (observed) contributions of the player's neighbors. Each experiment lasted for 10 rounds. In each round, every player had 10 points to allocate. A player can either keep a point, or put it into a local community pool. At the end of the round, a player's total points are the points they kept plus $40 \%$ of the points contributed by them and their neighbor set to the local community pool. All network topologies tested had uniform degree 5. Thus, if all nodes contributed 10 points to the local community pool, then each node would receive 24 points. At the other extreme, if all nodes contributed 0 to the pool, then each node would get only its 10 original points. Under this payoff structure there is a significant benefit to being in a highly-cooperative society.

In observations that span from disconnected cliques (high clustering) to random regular graphs (low clustering) Suri and Watts observe a race to nearly-complete defection. ${ }^{10}$ Suri and Watts write,

"In contrast with previous theoretical work, we found that network topology had no significant effect on average contributions. This result implies either that individuals are not conditional cooperators, or else that cooperation does not benefit from positive reinforcement between connected neighbors."

In follow-up tests conditional cooperation was observed, but the authors noted a qualitative symmetry: positive response to high-contributing neighbors did not outweigh negative response to low-contributing neighbors. Suri and Watts concluded that no effect like cooperative contagion seemed to spread beyond immediate contacts (regardless of network topology).

Our new view. Are the observations from (Suri \& Watts (2011)) truly incompatible with the message that clustered network topology benefits cooperation among conditional cooperators? Modeling the Suri and Watts experiments in our simple Shocks-of-Defection/Catastrophic-Collapse framework suggests that the lack of significant variation across topologies observed in (Suri \& Watts (2011)) is not at odds with the message that network clustering can positively impact cooperation.

In particular, we propose that the lack of significant variation observed across networks topologies tested in (Suri \& Watts (2011)) has another very plausible explanation not considered by Suri and Watts: their public-goods game induces a high percentage of initial defectors whose spatial placement (and total number) is not controlled under their experimental design, and the rules/user-interfaces of their game induce a high threshold for cooperation. Under our modeling framework, these factors force high liklihood of catastrophic collapse across all five topologies they test. Because cooperation has almost-completely collapsed in every topology, point contributions are practically indistinguishable. Additionally, our model predicts that random variation for a fixed network topology is also substantial (undermining the possibility of well-separated distributions).

We consider how to model the complicated public-goods game of Suri and Watts as a simple thresholdfor-cooperation game in a network. Our simple framework involves 4 inputs: a network, a fraction describing

\footnotetext{
${ }^{10}$ Almost all nodes contribute $0-2$ points out of 10 , with $80 \%+$ contributing 0 .
} 
the number of players who initially defect, a threshold for cooperation, and a sample size (how many different instances are measured for each topology).

- Networks: We use the five 24-node networks described explicitly in Figure 2 of (Suri \& Watts (2011)). Each node has degree 5.

- Fraction of players who initially defect: Figure 6 of (Suri \& Watts (2011)) shows the observed distribution of first round point contributions. We will assume that an initial contribution half or less of the possible points (0-5 out of 10) will be viewed as defection by neighbors. This classifies roughly $45 \%$ of players round- 1 contributions as defections. As in our computational experiments, Suri and Watts don't control the spatial placement of these human defectors. Unlike in our computational experiment (where a defection shock had a fixed size), $45 \%$ now represents the probability of selecting a human player who, confronted by the public-goods game of Suri and Watts, decides to start round 1 with a play that will be interpreted as a defection. Thus, we model each player (node) as binomial random variable with mean $45 \%$. We assume a shock duration of 1 time step. ${ }^{11}$

- Threshold for cooperation: Suri and Watts use a point-contribution system and aggregate player contributions: it is not immediately clear how to choose a simple threshold-for-cooperation to model their observations. We note, however, that all experiments of Suri and Watts end in what we consider catastrophic collapse of cooperation after 10 rounds. Is there a uniform threshold-for-cooperation ${ }^{12}$ that would cause this behavior given initial defection rate of $45 \%$ in the five tested networks from (Suri \& Watts (2011))?
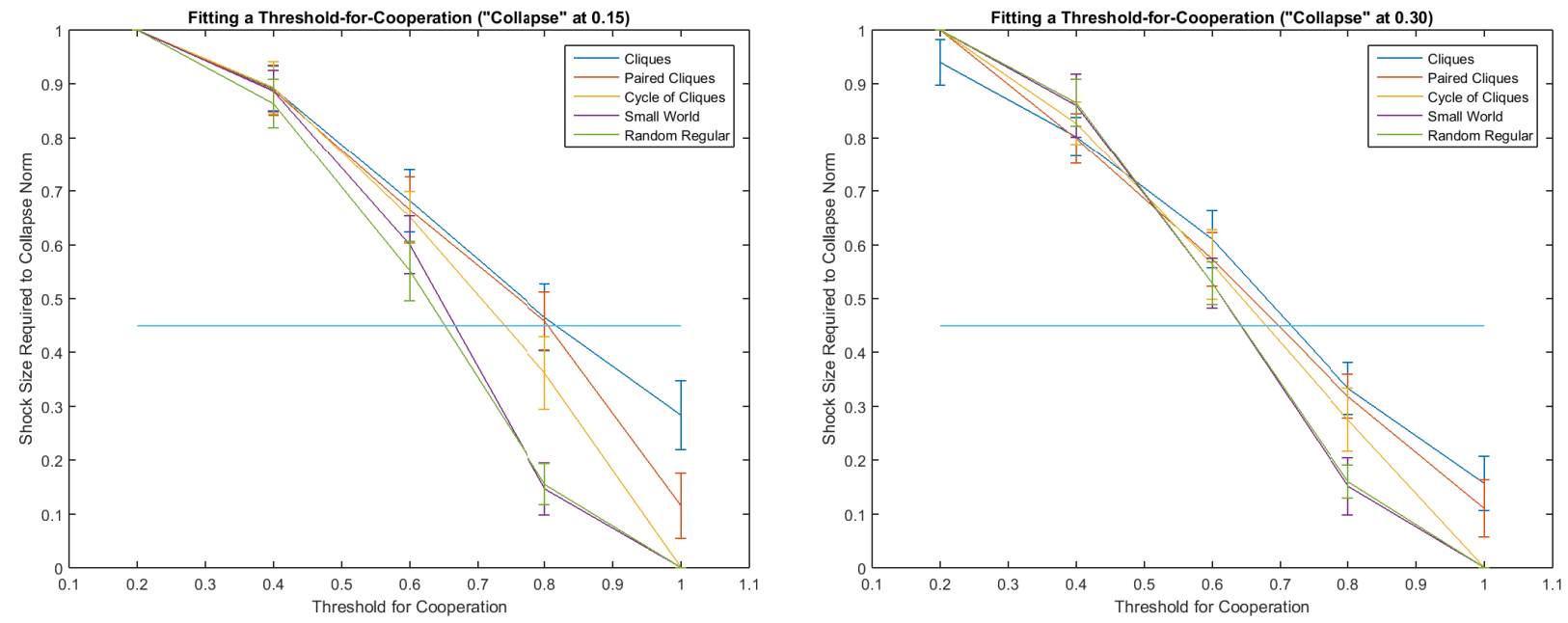

Figure 9: Fitting a Threshold-for-cooperation for catastrophic collapse across Suri and Watts topologies. The left and right panels are based on definitions of catastrophic collapse of $15 \%$ and $30 \%$ respectively. First-round defection of $45 \%$ is shown as a horizontal line.

Figure 9 depicts the size of shock required to cause catastrophic collapse as a function of the threshold for cooperation. Since each node has degree 5 in (Suri \& Watts (2011)), the thresholds that yield distinct spread behaviors will be $\{0.2,0.4,0.6,0.8,1\}$. All five topologies from (Suri \& Watts (2011)) are tested. The left and right panels are based on definitions of catastrophic collapse of $15 \%$ and $30 \%$ respectively.

\footnotetext{
${ }^{11}$ Immediately after round-1 play, we assume that individuals proceed as conditional cooperators.

${ }^{12}$ It is entirely plausible that thresholds are heterogeneous over the population of players, however we are curious whether even a very-simple uniform-threshold model can capture the all-networks-collapse outcome observed by Suri \& Watts (2011).
} 
We interpret our catastrophic collapse definitions from the previous section in terms of the small 24node networks of Suri and Watts. When catastrophic collapse is defined as at most $15 \%$ cooperation (corresponding to the left panel of Figure 9), at most 3 nodes of 24 will be contributing more than 6 points each at the end of 10 rounds. In the 24-node networks from (Suri \& Watts (2011)) nodes are arranged roughly in communities of 6 . Thus, at a high threshold for cooperation, a $15 \%$ definition of collapse does not register collapse in practice until 0 nodes of 24 are cooperating. When catastrophic collapse is defined as 30\% (corresponding to the right panel of Figure 9), at most 7 nodes (roughly one 6 -node community) will contribute more than 6 points each at the end of the game. In Figure 9, while some separation of topologies is observed when "catastrophic collapse" is defined as effectively-zero cooperation, there is almost no separation of topologies (regardless of threshold) when "catastrophic collapse" allows for at least one 6-node cluster to cooperate. Under the $30 \%$ definition, a first-round defection level of $45 \%$ is more than sufficient to consistently cause catastrophic collapse for all topologies when the threshold for cooperation is 0.8 or above. Thus, we choose to model the experiments of Suri and Watts using a threshold for cooperation of 0.8 (4 of 5 neighbors must collaborate for a node to choose cooperation).

- Sample Size: Suri and Watts give explicitly the number of realizations tested for each network topology (Cliques: 4, Paired Cliques: 3, Cycle Cliques: 8, Small World: 4, Random Regular: 4).

Given this simple model (threshold-for-cooperation of 4/5, initial defection rate with mean $45 \%$ ), we estimate the probability of catastrophic collapse to cooperation of $30 \%$ or less for each of the networks tested in (Suri \& Watts (2011)). Running our model 200 times, we also compute 2-sample-standard-deviation confidence intervals (approximately 95\%-confidence intervals) on the number of cooperating players predicted at the end of the game:

\begin{tabular}{|l|l|l|}
\hline $\begin{array}{l}\text { Network Topology } \\
\text { Initial Defection Rate of } 45 \%\end{array}$ & $\begin{array}{l}\text { Estimated Probability of } \\
\text { Final Cooperation } \leq 0.30\end{array}$ & $\begin{array}{l}\text { 2 Std. Dev.- confidence interval for } \\
\text { number of final cooperators (of 24) }\end{array}$ \\
\hline Cliques & $89.5 \%$ & $4.3(+/-9.2)$ \\
Paired Cliques & $91.5 \%$ & $4.1(+/-8.7)$ \\
Cycle Cliques & $94.0 \%$ & $1.7(+/-7.1)$ \\
Small World & $99.5 \%$ & $0.2(+/-3.6)$ \\
Random Regular & $99.5 \%$ & $0.1(+/-3.4)$ \\
\hline
\end{tabular}

As noted in the table above, our model predicts that at a $45 \%$ rate of initial defections, catastrophic collapse of cooperation is, by far, the most likely outcome for every network topology tested by Suri and Watts. This behavior is driven by two key features:

- Conditional cooperators who apply a high threshold for cooperation (4/5 in our simulation).

- A high fraction of round-1 defectors (mean $45 \%$ in our simulation) whose spatial placement is not controlled.

Further, our model produces confidence intervals for the final number of cooperators that overlap extensively. Because all topologies are pushed to states of catastrophic collapse of cooperation, levels of cooperation are naturally almost impossible to distinguish.

Thus, referring to the experiment replication Suri and Watts conduct for each topology, ${ }^{13}$ our simple model estimates the probability that Suri and Watts observe catastrophic collapse in every replication for every topology they test (assuming these are independent) to be roughly $28.8 \%$.

\footnotetext{
${ }^{13}$ Cliques: 4, Paired Cliques: 3, Cycle Cliques: 8, Small World: 4, Random Regular: 4. Notably, (Suri \& Watts (2011)) describes increasing the number of replications for Cycle Cliques topology due to an early sample deemed to be non-representative (the initial number of defectors was low, leading to much higher contributions throughout the 10-round game). Dynamically adjusting sample replication to damp rare events is statistically problematic.
} 
Both the high threshold for cooperation applied by players and the $45 \%$ fraction of round- 1 defectors can be understood as resulting from the specific reward structure and player-facing presentation of the publicgoods game in (Suri \& Watts (2011)). If a similar networked experiment were conducted for a public-goods game that naturally induced a lower percentage of initial defectors, or a lower threshold for cooperation, significant variation between topologies might very well be observed.

For example, our model predicts that even under the high threshold for cooperation of $4 / 5$ that players appear to apply in response to the game in (Suri \& Watts (2011)), if the initial fraction of plays viewed as defections could be reduced to $20 \%$, then a substantial difference could emerge between the tested topologies in terms of the likelihood of catastrophic collapse: Cliques: 11\%, Paired Cliques: $11.5 \%$, Cycle Cliques: 21.0\%, Small World: 64.5\%, Random Regular: 72.0\%. In human experiments, this might be explored by masking or inflating the true round-1 behavior of neighboring human players (as in the non-networked human experiments in (Rand et al. (2015))), or by redesigning the reward structure or presentation of the public-goods game.

\section{Results: Heterogeneous Moody Conditional Cooperation}

Our computational experiments in the previous sections investigate societies of uniform conditional cooperators (that is, we have assumed that the threshold applied by players, $h$, is uniform across the population $\left.^{14}\right)$. Thus, to explore the space of decision rules, in Figures $3-7$ we considered all possibilities for $h$ in $\{0.5,0.6,0.7,0.8,0.9\}$, and we noted a strong contrast with lowest values of $h$ (Figure 8).

Since the Moody Conditional Cooperation (MCC) Model has a more complex input (two thresholds per individual), and heterogeneity of player decision rules is a key finding of Grujić et al. (2010), it is now less obvious how we might systematically explore the parameter space of decision rules. In particular, even if a small number of player types are specified (fixing a $h_{v}^{c}$ and a $h_{v}^{d}$ for each such type), a very large number of distributions over player types of could be investigated. Thus, as in many computational studies, our investigation must necessarily be limited. We hope our work here will motivate subsequent studies.

We conduct experiments for two suites of distributions over moody conditional cooperator player types:

\begin{tabular}{l|c|c} 
Player Type & $h_{v}^{c}$ & $h_{v}^{d}$ \\
\hline Base Type & 0.6 & 0.9 \\
Generous Type & 0.1 & 0.4 \\
Stingy Type & 0.8 & 1.1
\end{tabular}

As a foundation, we consider a society in which $100 \%$ of individuals are moody conditional cooperators of the Base Type. Then we apply two treatments

- Distribution Suite 1: Adding Generous Moody Conditional Cooperators. For each $k \in$ $\{0,20,40,60,80,100\}, k \%$ of individuals of the Base Type are replaced with Generous Type individuals.

- Distribution Suite 2: Adding Stingy Moody Conditional Cooperators. For each $k \in$ $\{0,20,40,60,80,100\}, k \%$ of individuals of the Base Type are replaced with Stingy Type individuals.

In this way we obtain a total of 11 distributions, each over two types of conditional cooperators. The $k=0$ case gives a reference distribution common to both distribution suites. Within each suite, the distribution is adjusted gradually so that the behavior under different distributions will hopefully yield an interpret-able result. In all cases, the spatial placement of the types of players is randomized, e.g. for the $k=20$ case in Distribution Suite 1, exactly $0.2 *|V|$ individuals of the Generous Type are distributed uniformly at random among the $|V|$ nodes of $G$, and the remaining nodes are designated as Base Type.

\footnotetext{
${ }^{14}$ Of course, basic conditional cooperation could also be investigated when player thresholds are non-uniform.
} 


\subsection{Results: Adding Generous Moody Conditional Cooperators (MCCs)}

First we describe the experimental results for Distribution Suite 1. As in Section 3 for basic Conditional Cooperation, we perform tests starting from an initial society of 5 communities of 10 individuals each (Figure 10), an initial society of 20 communities of 10 individuals each (Figure 11), and a real network data set of 1,421 nodes based on co-membership in Norwegian public-limited boards from (Seierstad \& Opsahl (2011)) (Figure 12).
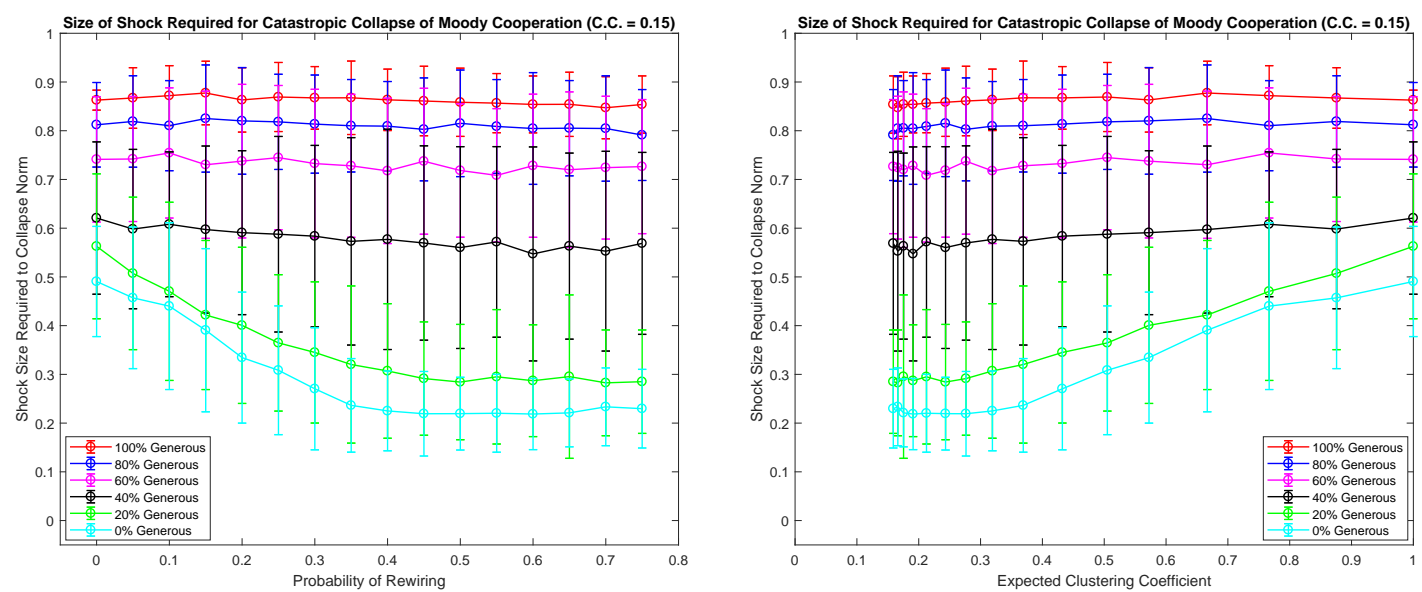

Figure 10: Adding Generous-Type Players Suppresses the Protective Effect of Clustering. Five initial communities of ten individuals each.
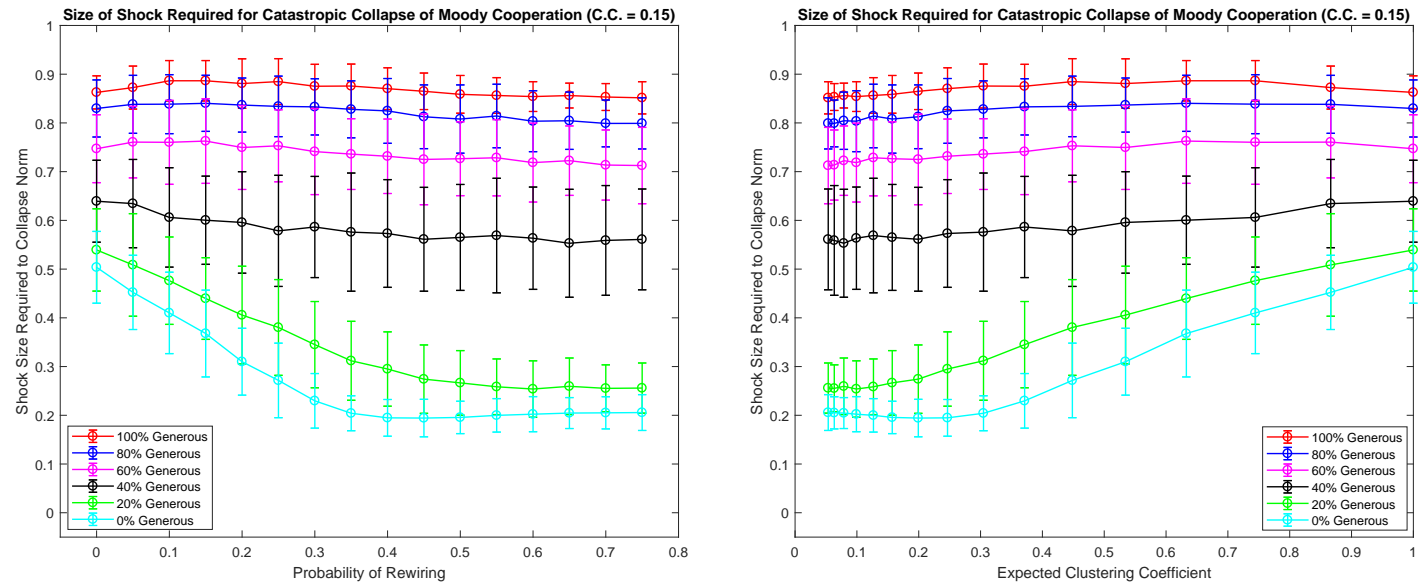

Figure 11: Adding Generous-Type Players Suppresses the Protective Effect of Clustering. Twenty initial communities of ten individuals each.

In the right panel of Figure 10, a strong protective effect of clustering is visible when the distribution of players is primarily Base-type MCCs (with $h_{v}^{c}=0.6$ and a $h_{v}^{d}=0.9$ ). This protective effect is still strongly apparent at $20 \%$ Generous-type MCCs, but appears to dissipate as an increasing number of Generous-type MCCs are added to the distribution. When the number of initial communities is increased to 20 (Figure 11) the results are remarkably consistent (though with notably lower variance).

Strikingly similar to our observations for basic conditional cooperation, the protective effect of clustering in Figures 10 and 11 appears to become active at a critical level that depends on the thresholds applied 

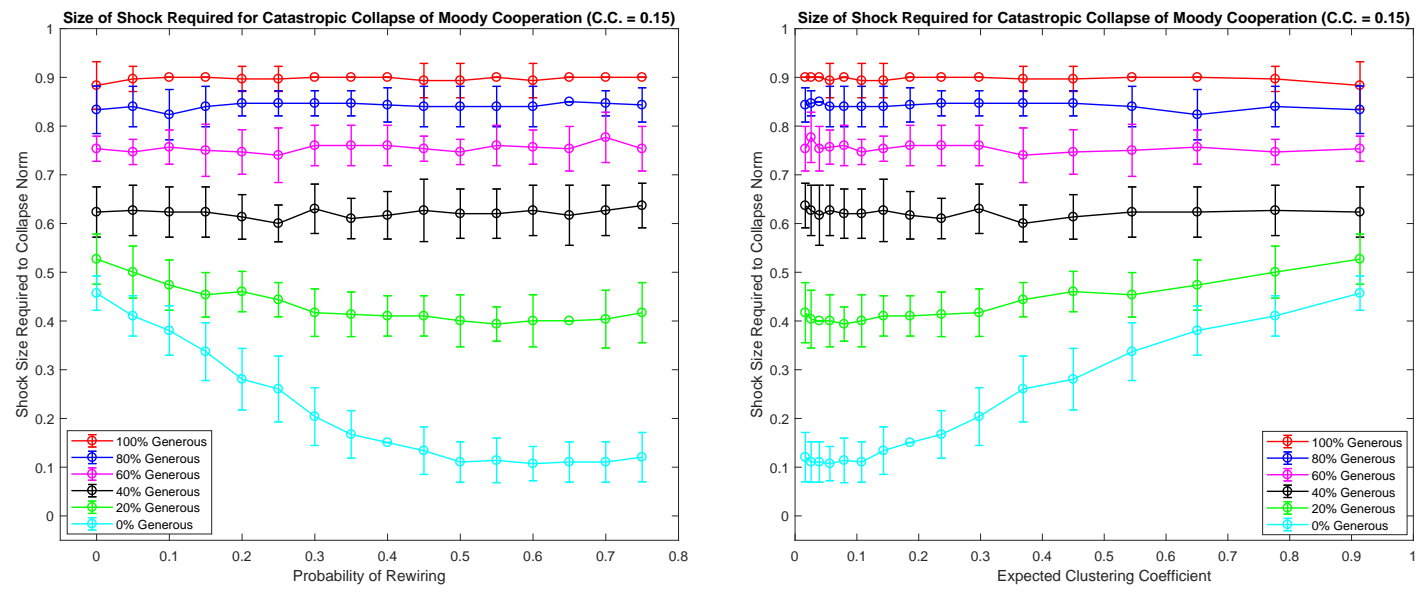

Figure 12: Adding Generous-Type Players Suppresses the Protective Effect of Clustering. Norwegian Coboard-membership Network (1,421 nodes).

by individuals. Namely, when more individuals apply higher cooperative and defective thresholds (aka, more MCCs are of the Base-type, rather than the Generous-type) this critical level appears to increase. Qualitatively, when more players respond as if the cost of cooperation is high, the protective effect of clustering becomes active at a higher critical level of clustering coefficient.

In the large real-network data set (Figure 12) again we observe a strong protective effect of clustering when the distribution of MCCs is entirely composed of Base-type MCCs, but in contrast with our small synthetic networks (in Figures 10 and 11), this effect has already become quite shallow by the time $20 \%$ Generous-type MCCs are added. In the Appendix we include an additional figure which shows that when Generous-type MCCs are added more gradually ( $5 \%$ at a time, rather than $20 \%$ at a time), many heterogeneous distributions over Base- and Generous-type MCCs do exhibit a protective effect of clustering.

\subsection{Results: Adding Stingy Moody Conditional Cooperators (MCCs)}

Next, we consider our experimental results for Distribution Suite 2. Note that the 0\%-Generous distribution from Suite 1 and the 0\%-Stingy distribution from Suite 2 are identical (all Base-type MCCs), so Figures 10 and 13 have a common reference distribution, Figures 11 and 14 have a common reference distribution, etc.

As Stingy-type MCCs are added to the distribution we obtain a series of heterogeneous player distributions that exhibit a very strong protective effect of clustering. As the player distribution becomes increasingly dominated by Stingy-type MCCs, in line with our previous observations, the critical level at which the protective effect of clustering becomes active shifts to the right (increases). For example, in the right-hand panel of Figure 14, at 40\%-Stingy-type MCCs the protective effect of clustering is first apparent around clustering coefficient 0.5 .

Across Figures 13 - 15 we also note that the magnitude of shock required to cause catastrophic collapse of cooperation is quite low. For example, in the right-hand panel of Figure 14, when even $20 \%$ of individuals are Stingy-type MCCs, if $30 \%$ of players initially defect, catastrophic collapse is predicted except at the very highest clustering coefficients (above 0.7).

Finally, we note that Figures 13 - 15 seem to indicate that the impact of adding Stingy-type MCCs is quite nonlinear: adding 60\% Stingy-type MCCs gives cooperation-collapse behavior that approaches the case when every single Base-type MCC is replaced by a Stingy-type MCC. At higher percentages of Stingy-type MCCs, the critical level of clustering required to activate a protective effect of clustering appears to be nearly 0.5 for our smaller synthetic networks (Figures 13 and 14).

In our computational experiments, we parameterized our Base-, Generous- and Stingy-type MCCs to make an initial exploration of behavior over the hetrogenous MCC parameter space. As in our interpretation of the 

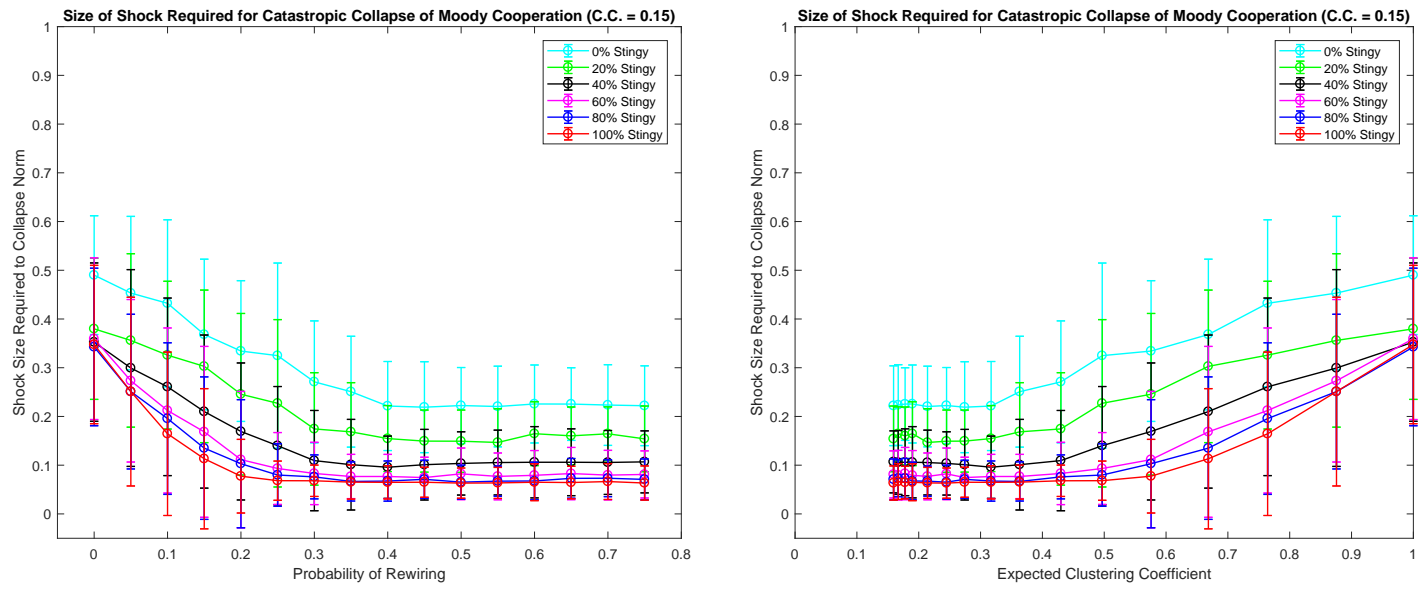

Figure 13: Adding Stingy Players Exposes the Protective Effect of Clustering, and Modest Shocks Cause Catastrophic Collapse of Cooperation. The 0\%-Stingy experiment in this figure coincides with the 0\%Generous experiment in Figure 10. Five initial communities of ten individuals each.
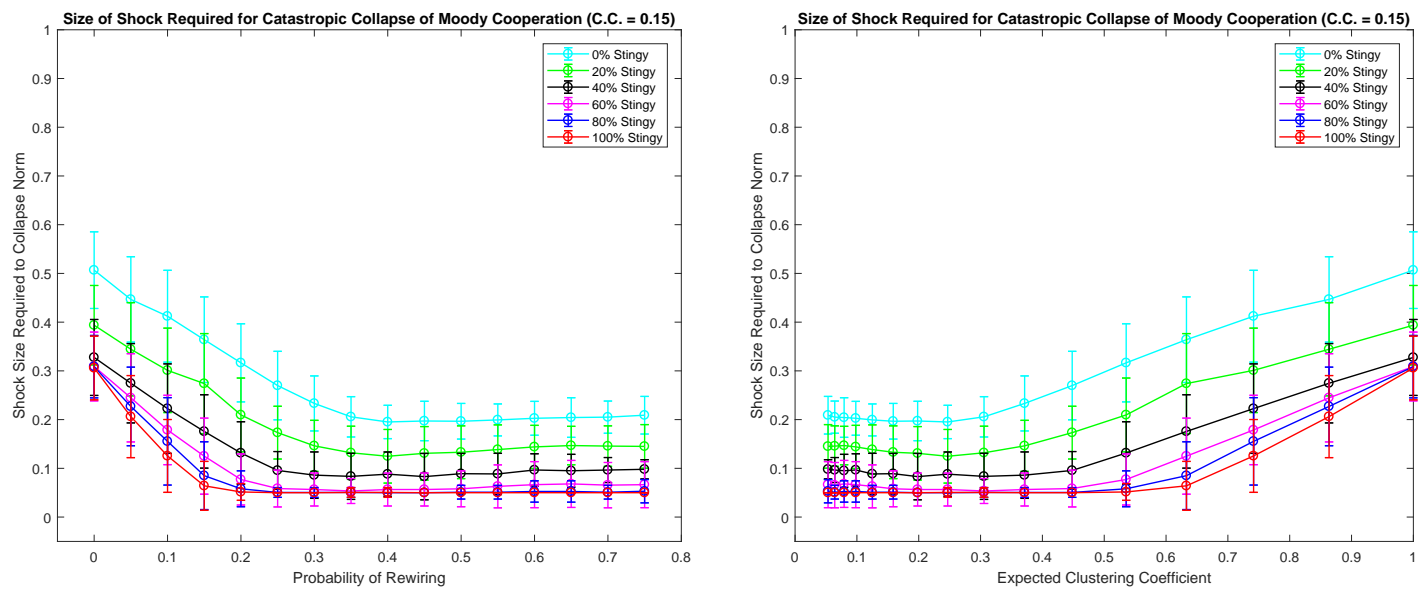

Figure 14: Adding Stingy Players Exposes the Protective Effect of Clustering, and Modest Shocks Cause Catastrophic Collapse of Cooperation. The 0\%-Stingy experiment in this figure coincides with the 0\%Generous experiment in Figure 11. Twenty initial communities of ten individuals each.

collapse of cooperation observed by Suri and Watts, we believe that the parameter values of decision rules applied by a population of players are a function of the presentation and reward structure of a networked game (this is also explicitly suggested for MCC by Grujić et al. (2014)). For Heterogeneous MCCs these parameters are the slopes and intercepts of plots like those depicted in Figure 1.

In particular, our framework suggests that human experiments in which

- Many players respond very generously despite the defection behavior of their neighbors

- Many players are very stingy and the clustering coefficients of networks explored are low ${ }^{15}$

- The percentage of round-1 defectors is large (relative to thresholds applied by players)

\footnotetext{
${ }^{15}$ We suspect that in more-complex shock scenarios, e.g. a series of on-going randomly distributed shocks that might describe persistent exploratory exploitation behavior, initial community size should also be modest, as in our computational experiments and the human experiments of Horita et al. (2017) in complete 4-node graphs.
} 

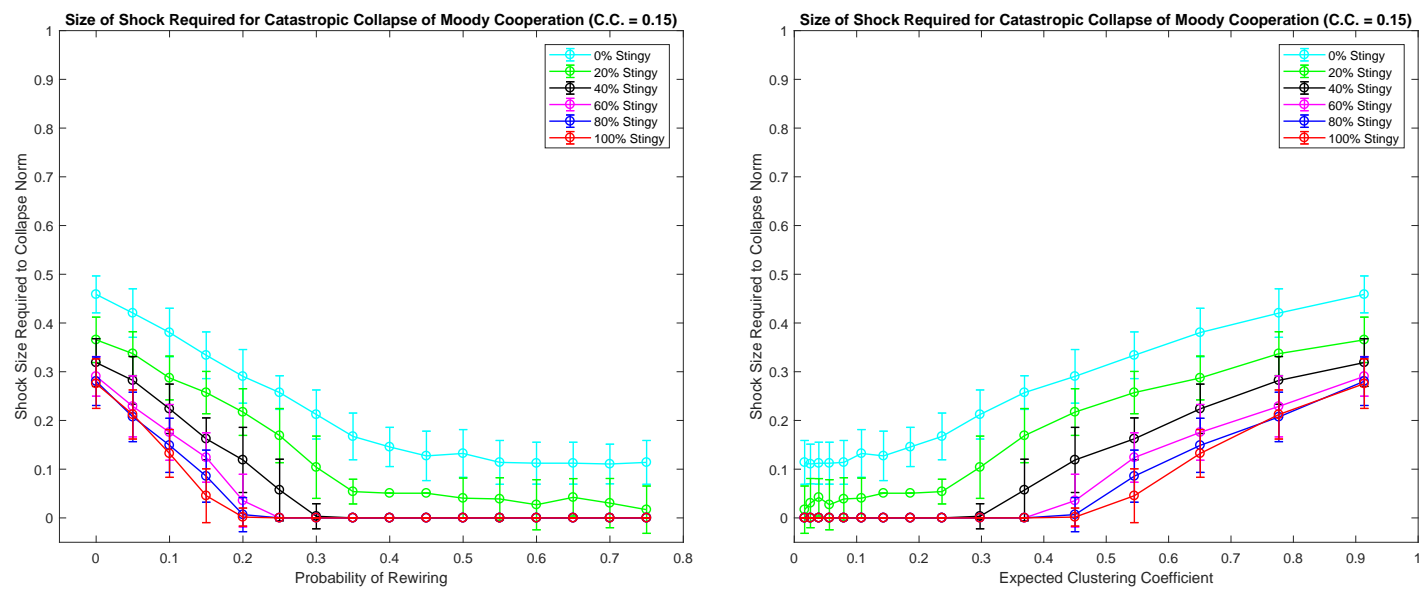

Figure 15: Adding Stingy Players Exposes the Protective Effect of Clustering, and Modest Shocks Cause Catastrophic Collapse of Cooperation. The 0\%-Stingy experiment in this figure coincides with the 0\%Generous experiment in Figure 12. Norwegian Co-board-membership Network (1,421 nodes).

cannot hope to detect a cooperation-promoting impact of network topology. Simply, such tests are being conducted in the wrong portion of the parameter space.

A Framework to Interpret Human Experiments for MCC. Some prominent human studies focused on Moody Conditional Cooperation ( $M C C$ ) are cited to argue that network topology doesn't impact rates of cooperation. We mention several such studies that are completely consistent with our framework.

Our observations may be useful in understanding the collapse of cooperation observed in studies like Grujić et al. (2010) where a full 24-40\% of players were classified as either pure defectors or mostly defectors (their Table 2). Such players behave similarly to our Stingy-type MCCs (if not even more stingily). Further, Grujić et al. (2010) document round-1 defection rates as high as 44-68\% (their Figure 1) and investigate only a degree-8 lattice (ego nodes with "Moore Neighborhoods") with clustering coefficient 0.43 . That is, Grujić et al. (2010) test in a portion of the Hetrogeneous MCC parameter space with a sizable population of stingy players, a large initial shock size, and low clustering coefficient (not obviously above the critical level of clustering we observe is required). Based on our Hetrogeneous MCC results, we would be extremely surprised if their experimental design resulted in something different than catastrophic collapse of cooperation. Indeed, they document cooperation collapse to roughly $22 \%$ (experiment 2 ), similar to an unstructured control treatment.

Gracia-Lázaro et al. (2012) announced emphatically that network topology is irrelevant for cooperation, titling their paper, "Heterogeneous networks do not promote cooperation when humans play a Prisoner's Dilemma." They drew this conclusion after testing only two different networks: one network with clustering coefficient 0 (a degree-4 lattice with periodic boundary conditions) and one network with clustering coefficient we estimate is at most at most 0.4 (a scale-free network with many long degree-2 paths). Gracia-Lázaro et al. (2012) observe similar collapse of cooperation in both networks. ${ }^{16}$

On the contrary, our results suggest that the observations from Gracia-Lázaro et al. (2012) are computationally predictable based on the parameters they observe for player response to their Prisoner's Dilemma

\footnotetext{
${ }^{16}$ Gracia-Lázaro et al. (2012) claim that the scale-free collapse should be particularly striking: they cite prior predictions that scale-free network topologies should promote cooperation. However, those prior predictions appear to be based on replicator dynamics (e.g. Santos \& Pacheco (2005)), a decision rule often studied in the physics literature that was already strongly discredited for repeated PD by Grujić et al. (2010). As our experimental results show, even adjusting the parameter values of a decision rule may impact whether a particular style of network is predicted to be cooperation promoting, e.g. our Figure 8 . That is, we don't believe that the scale-free network chosen in Grujić et al. (2010) constituted testing in a particularly-promising part of the space of networks given that players choose Moody Conditional Cooperation (and not replicator dynamics) in response to their PD game.
} 
Game. Of course, our evidence is from networks constructed by randomly rewiring dense local communities and not the precise networks tested in Grujić et al. (2010). Still, we must point out that Gracia-Lázaro et al. (2012) document a sizable 35-40\% shock of round-1 defections (their Figure 2), and large populations of verystingy players (roughly $40 \%$ based on the right-hand intercepts of panels A and B of their Figure 3). Based on the right-hand panels of our Figures 14 and 15, we would be strongly surprised if, with this combination of parameters for shock size, stingy fraction of the population, and clustering coefficient, Gracia-Lázaro et al. (2012) had observed something different than cooperation collapse in both networks they tested. Simply, our framework suggests that the empirical findings of Gracia-Lázaro et al. (2012) are very-plausibly compatible with the existence of a strong protective effect of clustering in other portions of the parameter space for networked Heterogeneous MCC.

In contrast, the Moody Conditional Cooperation human study of Horita et al. (2017) conducted all tests in groups of 4 (like the leftmost panel of our Figure 2 where the clustering coefficient is 1). Importantly, while levels of cooperation decreased over time, Horita et al. (2017) observed statistically higher rates of cooperation (for Prisoner's Dilemma) and more generous contributions (for a Public Goods Game) than under a mixed control condition for almost 20 consecutive rounds. Our computational framework suggests that there is no mystery here: Horita et al. (2017) conducted their tests in a portion of the Hetrogeneous MCC parameter space (highly-clustered networks) where our framework leads to a reasonable expectation that topology could positively impact cooperation.

Thus, we again suggest that experimenters interested in testing whether variation in network topology can impact levels of cooperation in human networked game play should engage in an advance stage of experimental design that measures and adjusts the game presentation and reward structure. Otherwise, lack of detection of an effect of network topology may be the computationally-predictable outcome of a particular experimental design, but nevertheless such an observation may be misinterpreted as a general message about the impotence of network structure to benefit levels of cooperation under a wider class of related games.

We close this section with a high-level comment. As in Subsection 3.3, our results for MCC Distribution Suite 1 appear to suggest that a search for a network-topology impact will be fruitless when many players behave as if the cost of cooperation is very low (or, symmetrically, as if the personal benefits they realize from acting as a cooperator, regardless of neighbor behavior, are very high). A subtle point here is that the monetary payoffs in many human game-play studies are usually quite modest: players may have quite variable perceptions of the value of these small financial rewards. Further, it seems plausible that some egoistic benefits a player accrues from feeling that they have behaved cooperatively (and thus are a "nice person," etc) can vary considerably. That is, suppose that highly-heterogeneous decision rules are truly unavoidable in existing-style laboratory experiments. If the uniformly-random spatial distribution of a sizable number of Generous-type players is truly unavoidable despite more strategic experimental design (and the effect of this is that network topology is irrelevant in all laboratory studies of existing styles), it seems essential to clarify whether the breadth of the distribution of decision rules observed in laboratory settings, including the "significant fraction of stubborn defectors and cooperators" (Grujić et al. (2010)), is truly indicative of breadth of human responses to higher-stakes cooperation vs. defection dilemmas outside of the laboratory. Namely, is the volume of the "tails" of pure cooperators and pure defectors that are spatially placed at random throughout a structured society really as large as it appears in relatively lowstakes laboratory games? Our study suggests that the "fatness" of such tails may reflect critically on whether the clustering ubiquitously observed in real social networks can be interpreted as utility-providing to societies that exhibit such clustered structure.

\section{Conclusion}

We propose a new view on how clustered social-network structure can benefit a society. While previous models focused on outbreaks of cooperation in an initially uncooperative network, both the prevalence of many large cooperatively-functioning human organizations and the frequent empirical observation that cooperation may spontaneously arise and stabilize in small dense communities lead us to consider an initial state where cooperation is widespread. Societies are at an advantage when they are able to maintain a basic level of 
cooperation despite occasional occurrences of pure defectors, transient experimentation with exploitation strategies, or large-scale hardships that may cause normally-cooperative players to defect for some period. We formalize this notion as avoiding catastrophic collapse of cooperation.

When individuals are conditional cooperators or moody conditional cooperators, a societal structure that retains a substantial "stable cooperative core" may accelerate regrowth of widespread cooperation after a cascade of defections. From a modeling perspective, if a stable cooperative core survives, the recovery of widespread mutually-beneficial cooperative norms need not rely as closely on rare coincidental acts of personal altruism. From a behavioral perspective, cooperation within a stable core may spill over to new groups of contacts (Cason et al. (2012); Fowler \& Christakis (2010)), or cooperative groups may slowly grow by including new members (Rand et al. (2015)). This "surviving foundation of cooperation" would appear even more advantageous under dynamic-network models where individuals can sever ties with defectors (on some time-scale). In contrast, a societal structure in which cooperation is driven to zero by milder shocks may be slower to regrow widespread cooperative norms, and thus experience a lower stream of benefits over time.

We observe computationally that as clustering decreases (as local links are randomly rewired), progressively smaller shocks of defection are sufficient to force the network into catastrophic collapse. The shock size required to force catastrophic collapse appears to depend in a surprisingly-smooth almost-linear manner on the clustering coefficient, and this was observed even in a sizable real-data example. We also observe that when players apply higher thresholds for cooperation (under basic Conditional Cooperation), or a sizable percentage of players are Stingy (under Moody Conditional Cooperation) this protective effect of clustering does not become active until an increasing critical value of clustering coefficient is reached. Additionally, the protective effect of clustering is suppressed when players behave as if the cost of cooperation is very low (under basic Conditional Cooperation, when players apply very low thresholds, and under Moody Conditional Cooperation when a sizable population of players are Generous).

Though our model gives a very coarse description of the networked public-goods game of Suri and Watts, ${ }^{17}$ we show that their apparently-counter-intuitive observations are compatible with our model. Lack of significant variation due to topology in their experiments can be understood as a function of the particular parameters of the public-goods game they designed. Because players respond to the public-goods game in (Suri \& Watts (2011)) with such a high level of defection in the first round, and demand such a high percentage of their neighbors to cooperate, our model predicts that catastrophic collapse is close-to-unavoidable regardless of topology. Thus, final levels of cooperative play are predicted to be very difficult to distinguish among topologies (as Suri and Watts found empirically).

Our computational observation that a critical level of clustering must be reached before a protective effect of clustering becomes active may also lend insight about why human experiments like Grujić et al. (2010) and Gracia-Lázaro et al. (2012) that search for contrast in long-term cooperation rates between a few low-clustering networks detect no impact of network topology.

We note that models like ours suggest how experimenters in the social sciences might refine networked public-goods games prior to running wide-scale experiments. The round- 1 behavior of human players, and how various point contributions are interpreted by neighbors, could be probed with artificial opponents in an experimental-design phase, avoiding large experiments that are unlikely to yield significant variation.

Last we comment on some limitations of the present study that we hope may motivate future work. While our choice of a uniformly-distributed defection shock in a static network is useful in interpreting the outcome of laboratory experiments in which the spatial placement of players is random and spatial structure is constant and forced by the experimenter, a richer attempt to describe the evolution of cooperation in clustered society could consider

- Network structures that can evolve on some time-scale: players might be allowed to periodically make or break links in response to defections of current neighbors, and possibly in response to information about cooperative behavior of near contacts.

\footnotetext{
${ }^{17}$ For example, we completely ignore the fine-scale distribution of points, and have no expectation that time steps in our model correspond meaningfully to the 10 rounds played by the human subjects.
} 
- Spatially-concentrated shocks of defection: our argument about temporary perturbations in cost-benefit calculations due to a large economic event (depression or famine) seem likely to exhibit some spatial structure. For example, an economic downturn may disproportionately impact low-income people, who may disproportionately be linked to other low-income people.

- An on-going "rain" of spontaneous random defection behaviors in the midst of a widely-cooperative society to model exploration/reward-probing behavior. Such a model seems strongly motivated by empirical observations of strategy exploration Traulsen et al. (2010).

These possible additional model features each suggest some interesting directions, though particularly in the first two cases, we expect that observations may be subtly influenced by interactions between the particular choice of how to capture the new model feature and the initial network structure. For example, a spatially-concentrated shock that is a star (an ego node and each of his neighbors) may lead to a different set of observations about the role of clustering than a spatially-concentrated shock that is a short random walk. Our caution here is informed by the network seeding literature: in some forms of networks quite naive strategies can seed large cascades while in other forms of networks the same strategy may have little effect. A responsible study of spatially-concentrated shocks would need to investigate a variety of methods for producing spatial concentration, and as such we leave this intriguing direction to future work.

Also, to focus on the effects of varying network structure, in this work we have ignored another key finding in the behavioral literature: the strong role of reputation and longer-term history of play in shaping decision rules applied by individuals (Weber (2006)), and how this may aid robustness of cooperative play in the presence of short-term defections that are perceived as non-malicious (Rand et al. (2015)). This combination of features may also lead to a strong model-based case that societies interested in retaining cooperation are well-served by maintaining short ties (for various models of reputation formation among neighbors).

\section{References}

Axelrod, R., \& Hamilton, W.D. (1981). The evolution of cooperation. Science, 1390-1396.

Cason, T.N., Savikhin, A.C., \& Sheremeta, R.M. (2012). Behavioral spillovers in coordination games. European economic review, 56(2), $233-245$.

Centola, D., \& Macy, M. (2007). Complex contagions and the weakness of long ties. American journal of sociology, 113(3), 702-734.

Fortunato, S. (2010). Community detection in graphs. Physics reports, 486(3-5), 75 - 174.

Fowler, J.H., \& Christakis, N. A. (2010). Cooperative behavior cascades in human social networks. Pnas, 107(12), 5334-5338.

Garcia, J.A., \& Vega-Redondo, F. (2015). Social cohesion and the evolution of altruism. Games and economic behavior, 92(July), 74-105.

Glance, N.S., \& Huberman, B.A. (1993). The outbreak of cooperation. The journal of mathematical sociology, 17(4), 281-302.

Gracia-Lázaro, C., Ferrer, A., Ruiz, G., Tarancón, A., Cuesta, J. A., Sánchez, A., \& Moreno, Y. (2012). Heterogeneous networks do not promote cooperation when humans play a prisoner's dilemma. Proceedings of the national academy of sciences, 109(32), 12922-12926.

Granovetter, M. (1978). Threshold models of collective behavior. The american journal of sociology, 83(6), $1420-1443$.

Granovetter, M.S. (1973). The Strength of Weak Ties. The american journal of sociology, 78(6), 1360-1380. 
Grujić, J., Gracia-Lázaro, C., Milinski, M., Semmann, D., Traulsen, A., Cuesta, J. A., Moreno, Y., \& Sánchez, A. (2014). A comparative analysis of spatial Prisoner's Dilemma experiments: Conditional cooperation and payoff irrelevance. Scientific reports, 4(Apr.), 4615.

Grujić, J., Fosco, C., Araujo, L., Cuesta, J.A., \& Sánchez, A. (2010). Social experiments in the mesoscale: Humans playing a spatial prisoner's dilemma. Plos one, 5(11), 1-9.

Horita, Y., Takezawa, M., Inukai, K., Kita, T., \& Masuda, N. (2017). Reinforcement learning accounts for moody conditional cooperation behavior: experimental results. Scientific reports, 7 .

Knez, M., \& Camerer, C. (2000). Increasing cooperation in prisoner's dilemmas by establishing a precedent of efficiency in coordination games. Organizational behavior and human decision processes, 82(2), 194 216.

Leskovec, J., Lang, K.J., \& Mahoney, M. (2010). Empirical comparison of algorithms for network community detection. Pages 631-640 of: Proceedings of the 19th international conference on world wide web. WWW '10. New York, NY, USA: ACM.

Nowak, M. (2006). Five rules for the evolution of cooperation. Science, 314(5805), 1560-1563.

Nowak, M.A., Bonhoeffer, S., \& May, R.M. (1994). Spatial games and the maintenance of cooperation. 91(11), 4877-4881.

Rand, David G., Nowak, Martin A., Fowler, James H., \& Christakis, Nicholas A. (2014). Static network structure can stabilize human cooperation. Proceedings of the national academy of sciences, 111(48), 17093-17098.

Rand, D.G., Fudenberg, D., \& Dreber, A. (2015). It's the thought that counts: The role of intentions in noisy repeated games. Scholarly articles. Harvard University Department of Economics.

Roca, Carlos P., Cuesta, José A., \& Sánchez, Angel. (2009). Evolutionary game theory: Temporal and spatial effects beyond replicator dynamics. Phys. life rev., 6(4), $208-249$.

Santos, F, \& Pacheco, J. (2005). Scale-free networks provide a unifying framework for the emergence of cooperation. Phys rev lett, Jan.

Seierstad, C., \& Opsahl, T. (2011). For the few not the many? the effects of affirmative action on presence, prominence, and social capital of women directors in norway. Scandinavian journal of management, 27(1), $44-54$.

Suri, S., \& Watts, D.J. (2011). Cooperation and contagion in web-based, networked public goods experiments. Plos one, 6(3), e16836.

Traulsen, A., Semmann, D., Sommerfeld, R.D., Krambeck, H., \& Milinski, M. (2010). Human strategy updating in evolutionary games. Proceedings of the national academy of sciences, 107(7), 2962-2966.

van Huyck, J., Battalio, R., \& Beil, R. (1990). Tacit coordination games, strategic uncertainty, and coordination failure. American economic review, 80(1), 234-48.

Watts, D. J., \& Strogatz, S. H. (1998). Collective dynamics of'small-world'networks. Nature, 393(6684), $409-10$.

Watts, D.J. (1999). Small worlds: The dynamics of networks between order and randomness. Princeton, NJ, USA: Princeton University Press.

Weber, R.A. (2006). Managing growth to achieve efficient coordination in large groups. American economic review, 96(1), 114-126. 


\section{Appendix}

\subsection{Adjusting Shock Duration: Basic Conditional-Cooperation Model}
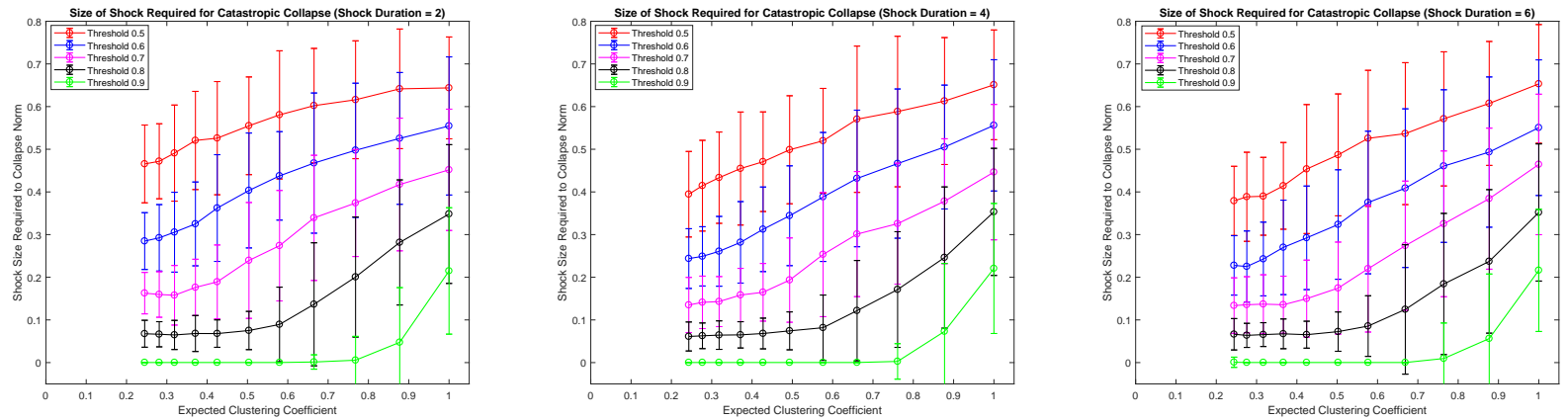

Figure 16: Catastrophic Collapse Behavior is Similar for Alternate Shock Duration. From left to right: duration $d=2$, duration $d=4$, duration $d=6$. Five initial communities of 10 individuals each (total society of 50 nodes).
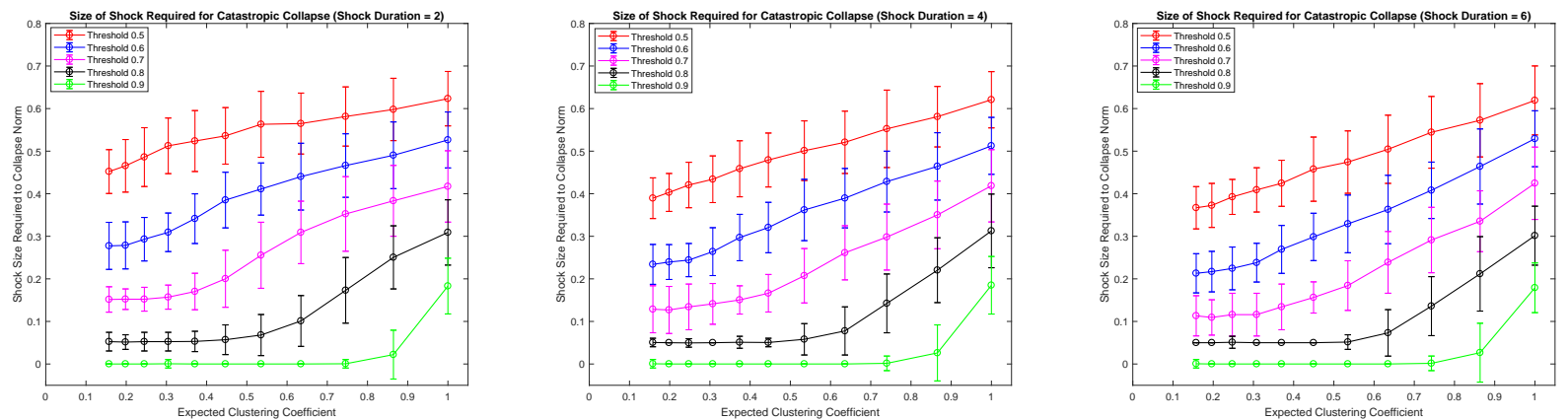

Figure 17: Catastrophic Collapse Behavior is Similar for Alternate Shock Duration. From left to right: duration $d=2$, duration $d=4$, duration $d=6$. Twenty initial communities of 10 individuals each (total society of 50 nodes).

\subsection{Rewired Dense Communities of Stochastic Size: Basic Conditional-Cooperation Model}

Figures 3, 4, and 5 are remarkably consistent despite a large difference in the relative size of a small community and the total society. In both previous cases, however, small community size is uniform. To verify that our results are not simply an artifact of our choice that each small initial community has the same size, we make several measurements when the sizes of the small communities in the initial society are chosen at random from a normal distribution.

Figures 18 - 20 depict the same computational experiment when the the initial small community sizes are chosen from a normal distribution, as noted in the caption of each figure. These figures are all based on a definition of catastrophic collapse of $15 \%$. The figures shown are quite typical of realizations we obtained when repeatedly realizing initial societies from the distributions described. We find these results to be remarkably consistent with those for uniform community sizes given in the main body of the paper. 

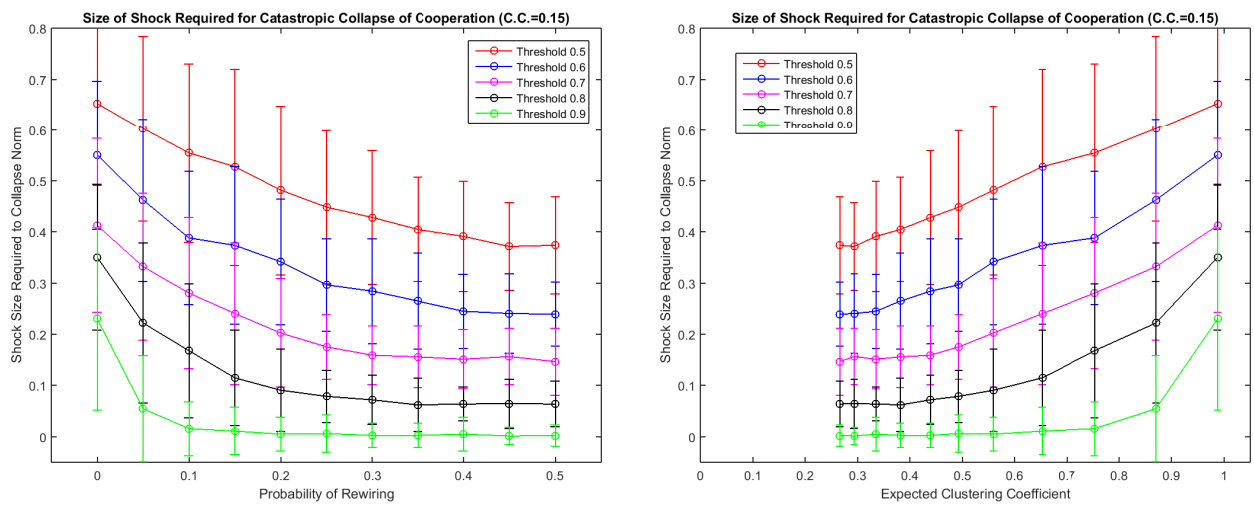

Figure 18: Ability to Withstand Defection Shocks vs. Rewiring (left) and Clustering Coefficient (right). Initial communities of mean size 10, standard deviation 5 (total society of 50 nodes).
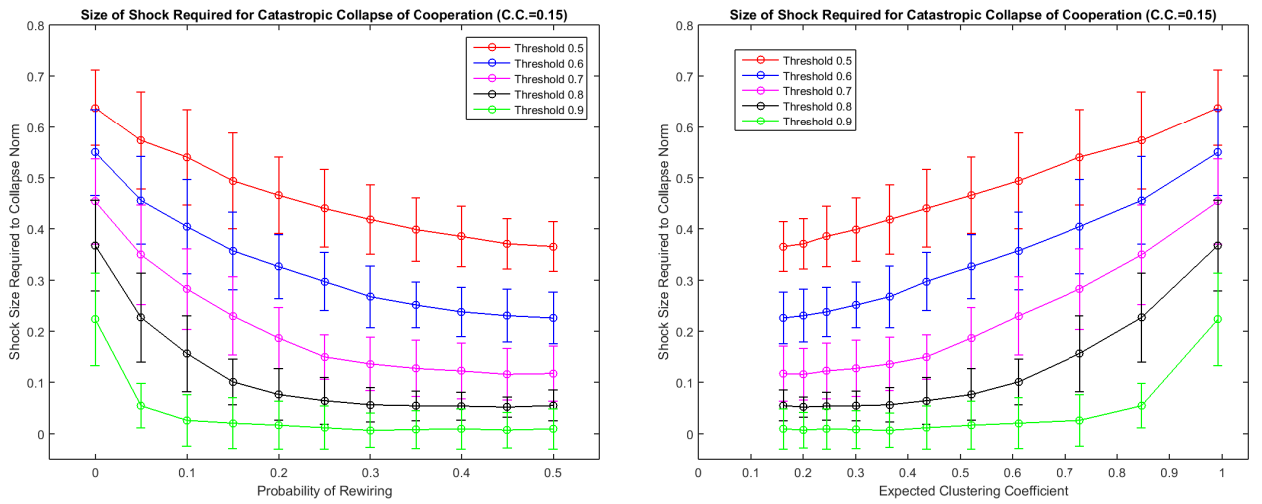

Figure 19: Ability to Withstand Defection Shocks vs. Rewiring (left) and Clustering Coefficient (right). Initial communities of mean size 10, standard deviation 5 (total society of 200 nodes).
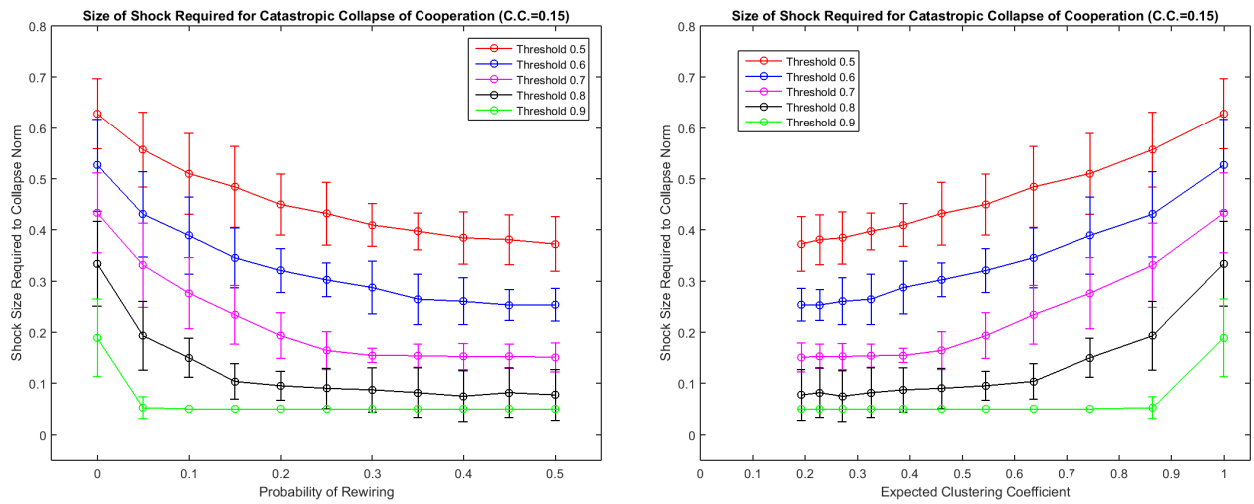

Figure 20: Ability to Withstand Defection Shocks vs. Rewiring (left) and Clustering Coefficient (right). Initial communities of mean size 20, standard deviation 5 (total society of 200 nodes). 


\subsection{Analogous Figures for "Catastrophic Collapse" at 30\%: Basic Conditional Cooperation}

Figures 21 and 22 are alternative versions of Figures 3 and 4, where the definition of catastrophic collapse is convergence to cooperation rate of $30 \%$ or less. Preserving the vertical axes from the earlier figures shows that the magnitude of the shock required to reach catastrophic collapse to $30 \%$ is somewhat lower for highly-clustered graphs (compared with collapse to 15\%), though almost the same for graphs that have been substantially rewired.

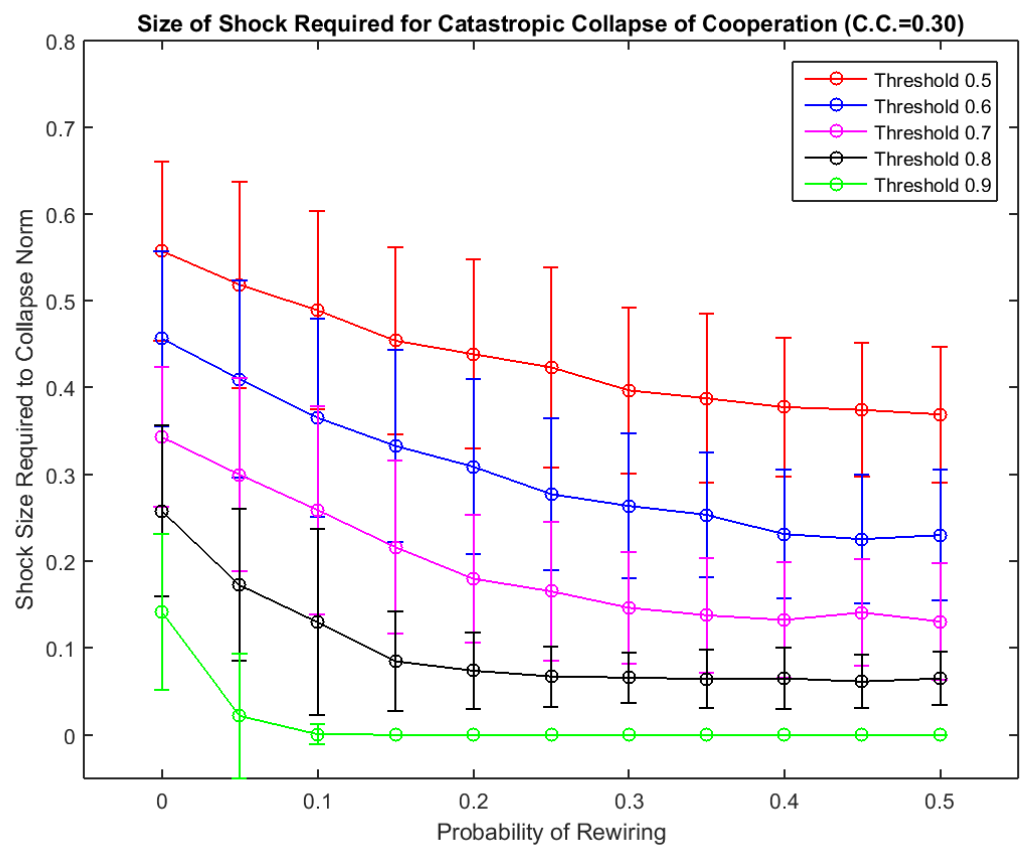

Figure 21: High Clustering Increases Ability to Withstand Defection Shocks. Five initial communities of ten individuals each.

Figure 23 below is an alternative versions of Figure 5 where the definition of catastrophic collapse is convergence to cooperation rate of $30 \%$ or less.

Figures $24-26$ are alternative versions of Figures $18-20$ where the definition of catastrophic collapse is convergence to cooperation rate of $30 \%$ or less. 


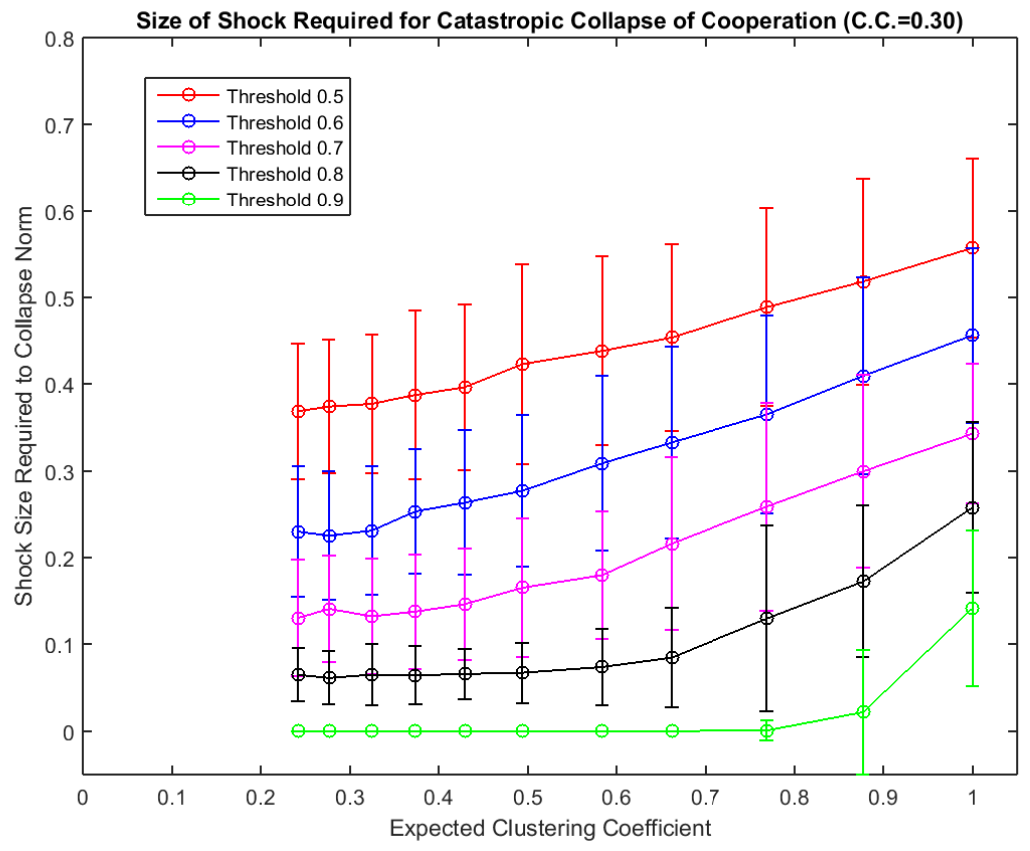

Figure 22: High Clustering Increases Ability to Withstand Defection Shock. Five initial communities of ten individuals each.
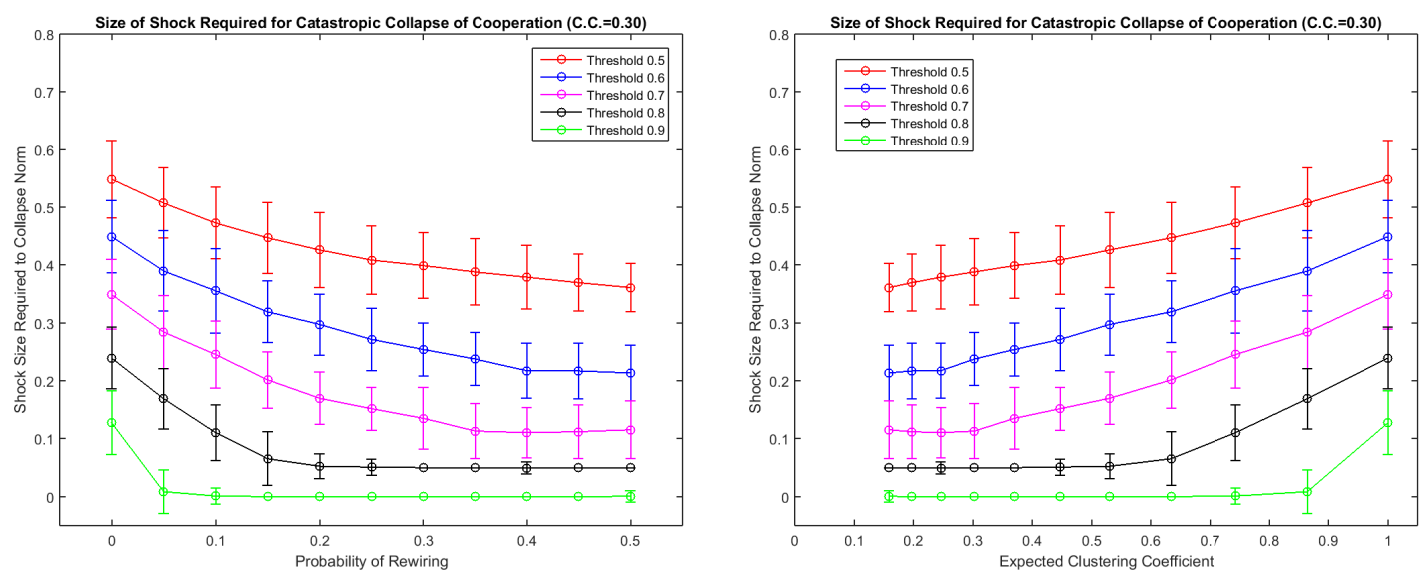

Figure 23: Ability to Withstand Defection Shocks vs. Rewiring (left) and Clustering Coefficient (right). Twenty initial communities of ten individuals each (total society of 200 nodes).

\subsection{Adding Generous MCCs more gradually: Heterogeneous Moody Conditional Cooperation}



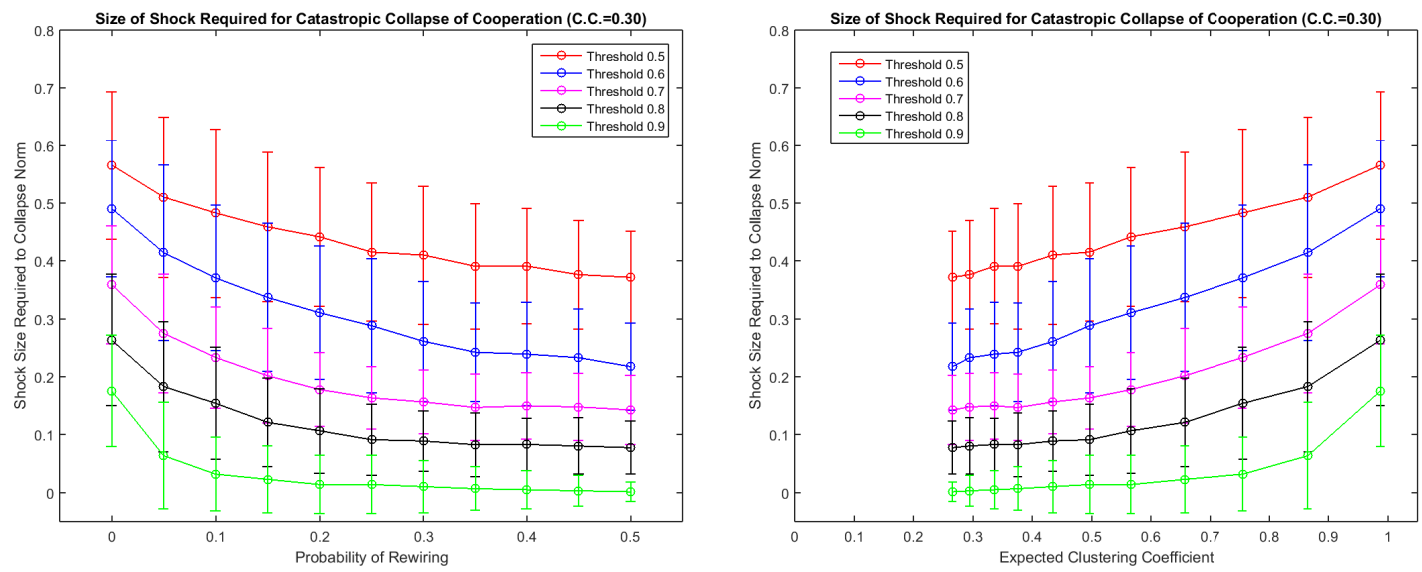

Figure 24: Ability to Withstand Defection Shocks vs. Rewiring (left) and Clustering Coefficient (right). Initial communities of mean size 10, standard deviation 5 (total society of 50 nodes).
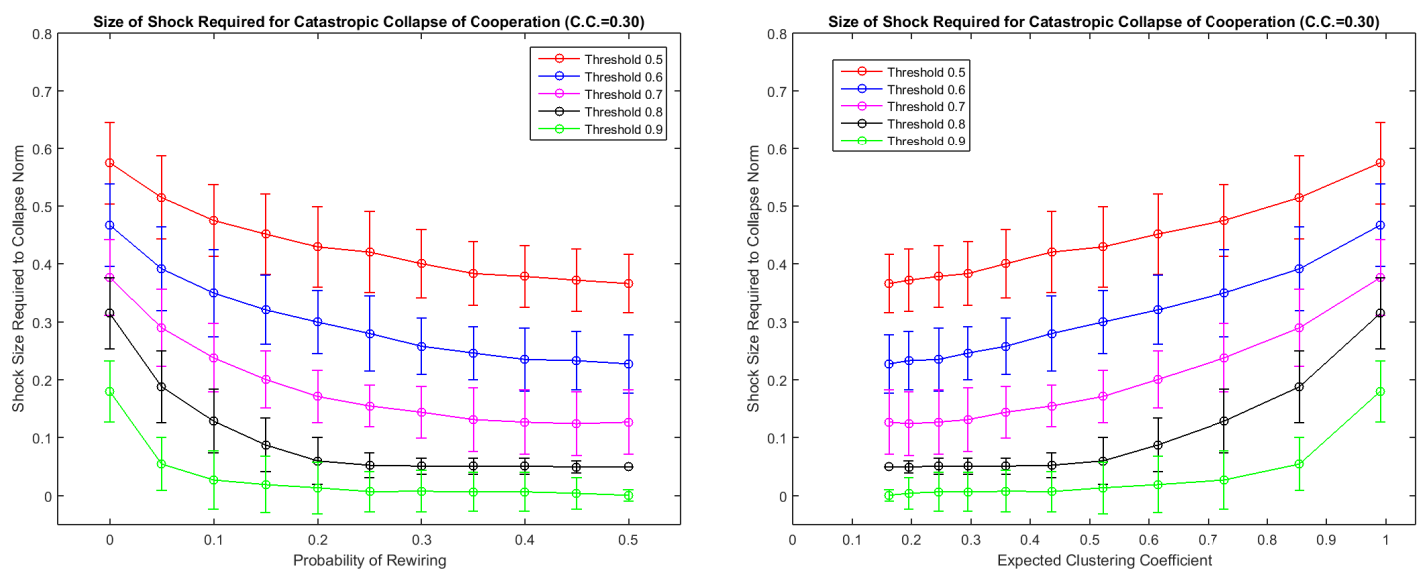

Figure 25: Ability to Withstand Defection Shocks vs. Rewiring (left) and Clustering Coefficient (right). Initial communities of mean size 10, standard deviation 5 (total society of 200 nodes). 

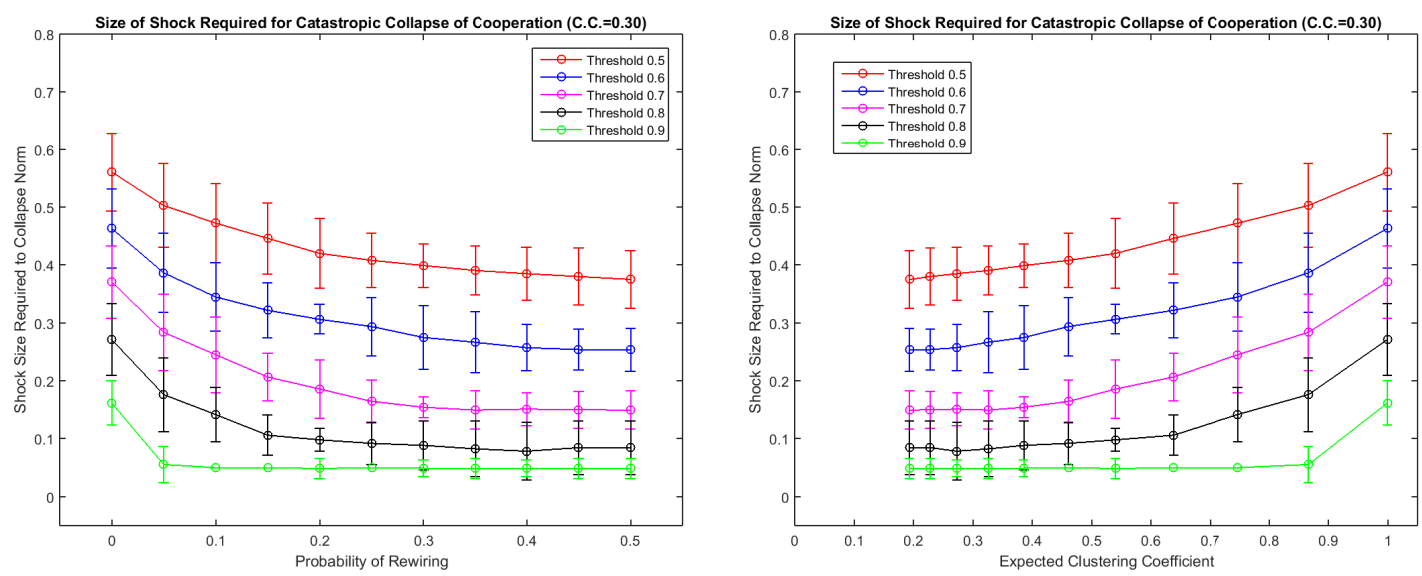

Figure 26: Ability to Withstand Defection Shocks vs. Rewiring (left) and Clustering Coefficient (right). Initial communities of mean size 20, standard deviation 5 (total society of 200 nodes).
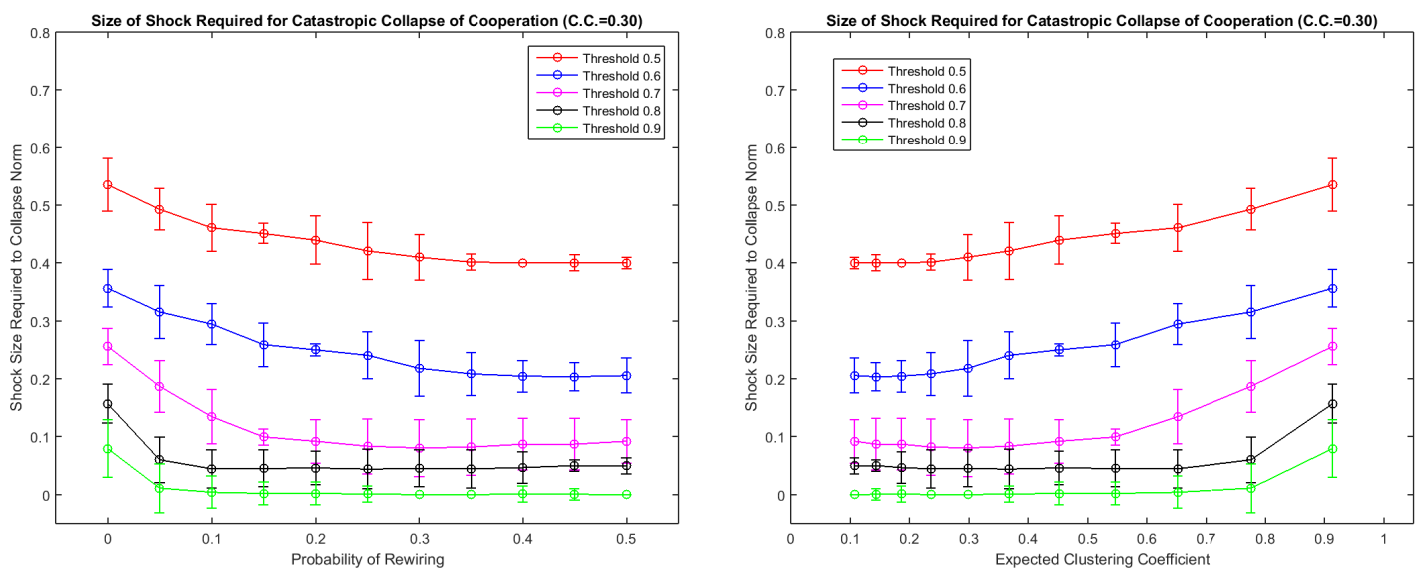

Figure 27: Ability to Withstand Defection Shocks vs. Rewiring (left) and Clustering Coefficient (right). Norwegian Co-board-membership Network (1,421 nodes). 

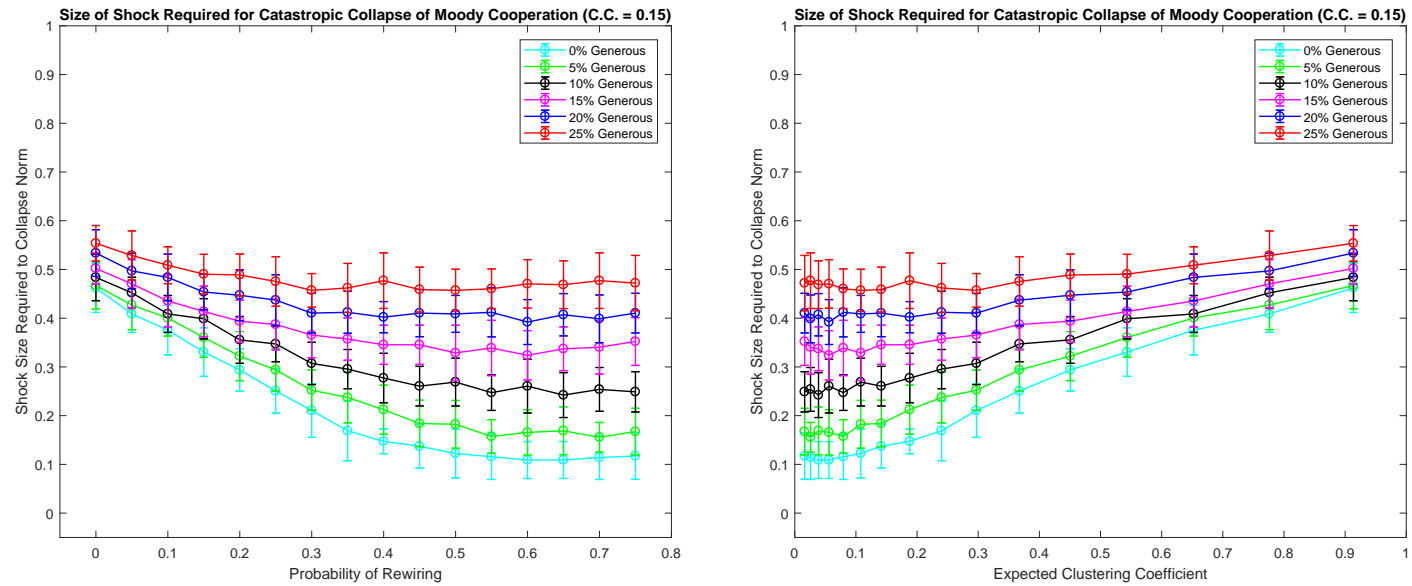

Figure 28: Adding Generous-Type Players Supresses the Protective Effect of Clustering. This figure examines the same experiment as Figure 11 but at increased resolution as generous players are added at $5 \%$ per distribution shown. For comparison, the 0\%-Generous case and 20\%-Generous case here are identical to those from Figure 11. Norwegian Co-board-membership Network (1,421 nodes). 